\title{
MASER OBSERVATIONS OF WESTERLUND 1 AND COMPREHENSIVE CONSIDERATIONS ON MASER PROPERTIES OF RED SUPERGIANTS ASSOCIATED WITH MASSIVE CLUSTERS
}

\author{
Thomas K. T. FoK ${ }^{1}$, Jun-IChi Nakashima ${ }^{1}$, Bosco H. K. Yung $^{1}$, Chin-Hao Hsia ${ }^{1}$, and Shuji Deguchi ${ }^{2}$ \\ ${ }^{1}$ Department of Physics, University of Hong Kong, Pokfulam Road, Hong Kong, China; junichi@ @ku.hk \\ ${ }^{2}$ Nobeyama Radio Observatory, National Astronomical Observatory of Japan, Minamimaki, Minamisaku, Nagano 384-1305, Japan \\ Received 2012 August 2; accepted 2012 September 26; published 2012 November 5
}

\begin{abstract}
We report the results of Australia Telescope Compact Array observations of the Westerlund 1 (Wd1) region in the $\mathrm{SiO} v=1, J=1-0$, and $\mathrm{H}_{2} \mathrm{O} 6_{16}-5_{23}$ maser lines, and we also report the analysis of maser properties of red supergiants (RSGs) associated with six massive clusters including Wd1. The primary purpose of this research is to explore possibilities of using maser emission for investigating the nature of massive clusters and associated RSGs. The $\mathrm{SiO} v=1, J=1-0$, and $\mathrm{H}_{2} \mathrm{O} 6_{16}-5_{23}$ maser lines are detected toward two of four known RSGs in Wd1. The large velocity ranges of maser emission are consistent with the RSG status. RSGs with maser emission tend to exhibit redder $\log \left(F_{21} / F_{12}\right)$ and [K-12.13] colors compared to RSGs with no maser emission. The mass-loss rates derived from dust radiative transfer modeling suggest that RSGs with maser emission tend to exhibit larger mass-loss rates compared to RSGs with no maser emission. In an extended sample of 57 RSGs in six massive clusters, detections in the $\mathrm{SiO}$ line tend to homogeneously distribute in absolute luminosity $L$, whereas those in the $\mathrm{H}_{2} \mathrm{O}$ line tend to distribute in a region with large $L$ values.
\end{abstract}

Key words: masers - open clusters and associations: individual (Westerlund 1) - stars: late-type - stars: mass-loss - supergiants - radio lines: stars

Online-only material: color figures

\section{INTRODUCTION}

Until now, about 200 red supergiants (RSGs) have been identified in the Galaxy (Levesque 2010), but a majority of known RSGs are isolated field stars, which pose difficulties in obtaining critical stellar parameters, such as distance, age, and luminosity. However, in the last several years some massive clusters harboring multiple RSGs have been found, including Westerlund 1 (Wd1; Mengel \& Tacconi-Garman 2007), Red Supergiant Cluster 1 (RSGC1; Figer et al. 2006), RSGC2 (Davies et al. 2007), RSGC3 (Alexander et al. 2009; Clark et al. 2009), RSGC4 (Negueruela et al. 2010b), and RSGC5 (Negueruela et al. 2011). These clusters that harbor multiple RSGs at a time are suitable sites for investigating the nature of RSGs, because RSGs in a single cluster share a coeval condition and the same distance.

It is notable that RSGs often exhibit maser emission of the $\mathrm{SiO}, \mathrm{H}_{2} \mathrm{O}$, and $\mathrm{OH}$ molecules. Since at the frequencies of these maser lines, interstellar extinction is negligible, maser emission potentially could be a useful tool for investigating RSGs hidden in the galactic plane. However, maser emission of RSGs embedded in massive clusters still has not been well studied. In order to explore possibilities of using maser emission for investigating massive clusters and embedded RSGs, we have performed maser searches toward five massive clusters, which are observable from the northern hemisphere, in the $\mathrm{SiO}(v=1$ and 2, $J=1-0)$ and $\mathrm{H}_{2} \mathrm{O}(22 \mathrm{GHz})$ maser lines (Nakashima \& Deguchi 2006; Deguchi et al. 2010), and until now roughly a dozen of maser sources of RSGs have been found in the five massive stellar clusters. In addition, the results of an $\mathrm{SiO}$ maser survey of Galactic RSGs in the SiO $v=1, J=2-1$ line have been recently reported (Verheyen et al. 2012).

In this paper, we report the results of interferometric maser observations using the Australia Telescope Compact Array (ATCA) toward the massive cluster $\mathrm{Wd} 1$ in the $\mathrm{SiO}$ and $\mathrm{H}_{2} \mathrm{O}$ maser lines at $43 \mathrm{GHz}$ and $22 \mathrm{GHz}$, respectively. The purposes of this research are (1) to search $\mathrm{SiO}$ and $\mathrm{H}_{2} \mathrm{O}$ maser emission toward the $\mathrm{Wd} 1$ region and (2) to explore possibilities of using maser emission for investigating the nature of massive clusters and associated RSGs. To clarify the maser properties of RSGs in massive clusters, we analyze the previous data of maser observations of massive clusters, in conjunction with the present observation of $\mathrm{Wd} 1$.

The structure of this paper is as follows. In Section 2, we describe the details of the observations of Wd1. In Section 3, we summarize the results of $\mathrm{SiO}$ and $\mathrm{H}_{2} \mathrm{O}$ maser observations of Wd1. In Section 4, we discuss the velocity information derived by the present observations. In Section 5, we discuss the infrared properties of RSGs in Wd1 and other massive clusters. Finally, the main results of the present research are summarized in Section 6.

\section{OBSERVATIONS AND DATA REDUCTIONS}

The radio interferometric observation in the $\mathrm{SiO} v=1$, $J=1-0$ line toward the $\mathrm{Wd} 1$ region was made with ATCA on 2008 November 20 and 21 (project code: C1796, PI: J. Nakashima). Since the angular size of the $\mathrm{Wd} 1$ region $\left(2^{\prime} \times 4^{\prime}\right.$; Borgman et al. 1970) is much larger than that of a single field of view (FOV) of an ATCA $22 \mathrm{~m}$ antenna at the observing frequency of $43 \mathrm{GHz}$ (half-power beam width, HPBW 66"), we observed eight FOVs with different center positions to cover all RSGs and RSG candidates in Wd1 (center positions of observed FOVs are summarized in Table 1). In Wd1, Mengel \& Tacconi-Garman (2007) have identified four RSGs (W 20, W 75, W 26, and W 237) through their near-infrared spectroscopy, and all the four known RSGs were covered in the present observation (coordinate values of the known RSGs are summarized in Table 2). In addition to four known RSGs, to make doubly sure, a majority of $K$-band bright stars in the Wd1 region (i.e., possible 


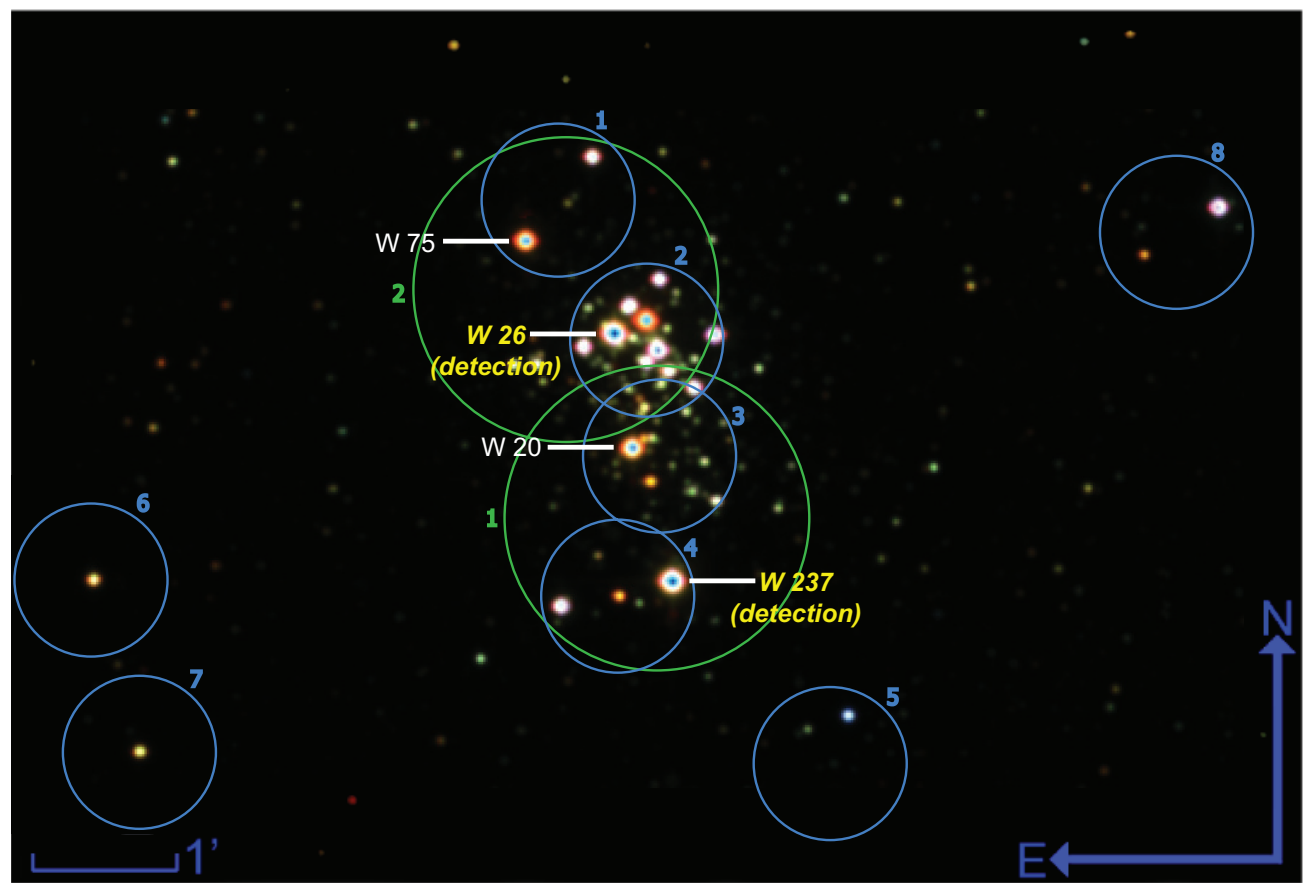

Figure 1. Positions of FOVs for the $\mathrm{SiO}$ and $\mathrm{H}_{2} \mathrm{O}$ maser observations at $43 \mathrm{GHz}$ and $22 \mathrm{GHz}$, respectively, superimposed on the $J, H$, and $K$ bands color composite 2MASS image (image size is $8^{\prime} .8 \times 5.9$; north is up, east is left). The blue and green circles represent FOVs for the $\mathrm{SiO}^{\prime}$ and $\mathrm{H}_{2} \mathrm{O}$ observations, respectively. The diameters of the blue and green circles (i.e., HPBW of an ATCA $22 \mathrm{~m}$ antenna) are 66" and 126", respectively. Each FOV is numbered according to Table 1. Known RSGs with no maser emission are labeled with object names in white color. RSGs detected in the $\mathrm{SiO}$ and $\mathrm{H}_{2} \mathrm{O}$ maser lines are labeled with object names in yellow color.

(A color version of this figure is available in the online journal.)

Table 1

Observational Parameters

\begin{tabular}{|c|c|c|c|c|c|}
\hline \multirow{2}{*}{$\begin{array}{l}\text { Position of } \\
\text { Observation }\end{array}$} & \multicolumn{2}{|c|}{ Center Position } & \multirow{2}{*}{$\begin{array}{c}\text { Integration Time } \\
\text { (hr) }\end{array}$} & \multirow[t]{2}{*}{ Beam Size $^{\mathrm{a}}$} & \multirow[t]{2}{*}{ P.A. $^{a}$} \\
\hline & R.A. (J2000.0) & Decl. (J2000.0) & & & \\
\hline \multicolumn{6}{|c|}{$\mathrm{SiO}$ Observation } \\
\hline Position 1 & $16: 47: 07.65$ & $-45: 49: 39.3$ & 0.65 & $5^{\prime \prime} .2 \times 2 . .5$ & 5.6 \\
\hline Position 2 & $16: 47: 04.20$ & $-45: 50: 38.3$ & 0.99 & $5^{\prime \prime} .1 \times 2$ 2.6 6 & $2: 7$ \\
\hline Position 3 & $16: 47: 03.62$ & $-45: 51: 26.3$ & 0.99 & $5^{\prime \prime} 0 \times 2.6$ & 4.7 \\
\hline Position 4 & $16: 47: 05.34$ & $-45: 52: 24.3$ & 0.50 & $8^{\prime \prime} .1 \times 2 . .2$ & 12.5 \\
\hline Position 5 & $16: 46: 56.82$ & $-45: 53: 33.2$ & 0.50 & $5^{\prime \prime} .9 \times 2 . .4$ & 14.7 \\
\hline Position 6 & $16: 47: 26.12$ & $-45: 52: 17.3$ & 0.50 & $8^{\prime \prime} .5 \times 2 . ! 2$ & 5.3 \\
\hline Position 7 & $16: 47: 24.31$ & $-45: 53: 29.3$ & 0.50 & $8^{\prime \prime} .3 \times 2 . .2$ & 9.3 \\
\hline Position 8 & $16: 46: 43.06$ & $-45: 49: 53.0$ & 0.50 & $5^{\prime \prime} .8 \times 2 . .4$ & $16: 6$ \\
\hline \multicolumn{6}{|c|}{$\mathrm{H}_{2} \mathrm{O}$ Observation } \\
\hline Position 1 & $16: 47: 03.90$ & $-45: 51: 51.4$ & 2.03 & $0^{\prime \prime} .9 \times 0^{\prime \prime} .4$ & -40.6 \\
\hline Position 2 & $16: 47: 07.20$ & $-45: 50: 17.5$ & 0.58 & $1^{\prime \prime} .7 \times 0 . .3$ & -33.7 \\
\hline
\end{tabular}

Note. ${ }^{\text {a }}$ Parameters of synthesized beams.

Table 2

List of Known RSGs in Westerlund 1

\begin{tabular}{lccc}
\hline \hline Source & 2MASS & \multicolumn{2}{c}{ 2MASS Position } \\
\cline { 3 - 4 } Name & Name & R.A. (J2000.0) & Decl. (J2000.0) \\
\hline W 237 & J16470309-4552189 & $16: 47: 03.09$ & $-45: 52: 18.9$ \\
W 26 & J16470540-4550367 & $16: 47: 05.54$ & $-45: 50: 36.9$ \\
W 20 & J16470468-4551238 & $16: 47: 04.69$ & $-45: 51: 23.9$ \\
W 75 & J16470892-4549585 & $16: 47: 08.93$ & $-45: 49: 58.6$ \\
\hline
\end{tabular}

mass-losing evolved stars) are covered with eight FOVs. In Figure 1, we present the locations of eight observed FOVs (see the blue circles in Figure 1) and four known RSGs.
The weather condition was stable throughout the entire observation run. The observation used the EW352/367 array configuration consisting of six $22 \mathrm{~m}$ antennas. The baseline lengths ranged from 50 to $4400 \mathrm{~m}$. The rest frequency of the $\mathrm{SiO} v=1, J=1-0$ line $(43.122090 \mathrm{GHz})$ was centered at a correlator window with a bandwidth of $32 \mathrm{MHz}$. The frequency coverage was ranged from 43.148 to $43.116 \mathrm{GHz}$. The 256 channel correlator gave the effective velocity coverage and resolution of $222.6 \mathrm{~km} \mathrm{~s}^{-1}$ and $0.869 \mathrm{~km} \mathrm{~s}^{-1}$, respectively. The quasar 1253-055 (3C279) was observed at the beginning of the observation for bandpass and flux calibrations. A nearby strong $\mathrm{SiO}$ maser source, IRAS 16105-4205 was also observed at the beginning for checking reliability of the system (the $\mathrm{SiO} v=1$, 
$J=1-0$ line is detected for the first time toward this object, even though the strong $v=1, J=2-1$ line was previously detected; Haikala et al. 1994, see Appendix A for details). The resulting uncertainty of the absolute flux density is roughly within $15 \%-20 \%$. Observations were made in a snapshot mode, which observed eight FOVs in turn. The observation was periodically interleaved with the nearby gain calibrators, 1646-50, to track the phase variations over time (the phase calibrator was observed for 3 minutes every roughly 30 minutes). After all positions were observed, the array slewed to the first position to loop again. At least three loops were repeated for each position throughout the whole observing session. The on-source integration time at each FOV was 10 minutes in each single loop except for positions 2 and 3, which took 15 minutes each (this is because positions 2 and 3 are the most crowded region in $\mathrm{Wd} 1$, a better signalto-noise ratio helps to avoid confusion in source identification). Total integration time on position 1 , positions $2-3$, and positions 4-8 were $0.7,1.0$, and $0.5 \mathrm{hr}$, respectively. And another $1.6 \mathrm{hr}$ were used for calibrations. Visibility data were calibrated using MIRIAD (Sault et al. 1995), following a standard calibration procedure. Then we applied a self-calibration technique using the MIRIAD task, selfcal to enhance the signal-to-noise ratio. Image processing of the data was also performed with MIRIAD by applying robust weighting, which compromises between the natural and uniform weighting (we applied "robust = 0.5 "). As we observed different FOVs in a snapshot mode, the $u-v$ coverages are different from FOV to FOV. Therefore, synthesized beams at each FOV have slightly different sizes and position angles; the information about synthesized beam sizes is summarized in Table 1 (note that the averaged synthesized beam size is $6.5 \times 2$.'4). We checked the detection of the continuum emission by integrating over all emission-free channels (the integration range is about $25 \mathrm{MHz}$ ), but did not detect it. The upper limit is $1.5 \times 10^{-3} \mathrm{Jy} \mathrm{beam}^{-1}$.

Archival ATCA data of a previous observation in the $\mathrm{H}_{2} \mathrm{O}$ $6_{16}-5_{23}$ line toward Wd1 (project code: C1619, PI : Sean Dougherty ${ }^{3}$ ) were used for the present analysis. The $\mathrm{H}_{2} \mathrm{O}$ maser observation was made from 2006 December 6 to December 10 using the 6A array configuration. The six antennas form baselines ranged from 340 to $5900 \mathrm{~m}$. The observed $\mathrm{H}_{2} \mathrm{O}$ line at $22.2351204 \mathrm{GHz}$ is centered at a correlator window with a bandwidth of $16 \mathrm{MHz}$. The frequency coverage is ranged from $22.243 \mathrm{GHz}$ to $22.227 \mathrm{GHz}$. The 512 channel correlator gave an effective velocity coverage and resolution of $215.7 \mathrm{~km} \mathrm{~s}^{-1}$ and $0.421 \mathrm{~km} \mathrm{~s}^{-1}$, respectively. The HPBW of a single antenna is $126^{\prime \prime}$ at the observing frequency. With this beam size of a single antenna, almost the entire cluster region was covered with only two FOVs. We present the locations of observed FOVs in Figure 1 (see the green circles) and Table 1. The observation was made in a snapshot mode as well as the $\mathrm{SiO}$ maser observation. A nearby phase calibrator, 1646-50, was observed every 10 minutes. The flux calibrator used was 1253-055 (3C279). Calibration and imaging of the data were performed using MIRIAD following the same methodology as adopted for the $\mathrm{SiO}$ data. We applied robust weighting (robust $=0.5$ ) giving an averaged synthesized beam size of $1^{\prime \prime} .3 \times 0.4$. We did not detect any continuum emission (integration range is roughly $10 \mathrm{MHz}$ ); the upper limit is $2.4 \times 10^{-3} \mathrm{Jy} \mathrm{beam}^{-1}$.

\footnotetext{
3 The $\mathrm{OH} 1612 \mathrm{MHz}$ line was also observed in this project, and the emission seems to be detected toward Wd1 according to our brief inspection. However, as the data quality is not good enough for further analysis, we do not use it in the present research.
}

\section{RESULTS OF MASER OBSERVATIONS OF WESTERLUND 1}

\subsection{Sources Identification}

First of all, we briefly describe how we identified stellar maser sources in the data cubes of the present observation. Source identification processes are common in both $\mathrm{SiO}$ and $\mathrm{H}_{2} \mathrm{O}$ observations. We first sorted out all emission features detected above a $3 \sigma$ level in each velocity channel of calibrated data cubes, and obtained the coordinate values of each emission feature by fitting a two-dimensional Gaussian function using a MIRIAD task imfit. At this stage, roughly 10 emission sources are selected as possible detections in each data set of the $\mathrm{SiO}$ and $\mathrm{H}_{2} \mathrm{O}$ observations. Then, we excluded artificial features in the following procedure.

Generally speaking, true maser lines exhibit a line width larger than, at least, $1-2 \mathrm{~km} \mathrm{~s}^{-1}$, which is equivalent to 2 and 3 channels at $43 \mathrm{GHz}$ and $22 \mathrm{GHz}$, respectively (velocity resolutions of the present observation at $43 \mathrm{GHz}$ and $22 \mathrm{GHz}$ are $0.869 \mathrm{~km} \mathrm{~s}^{-1}$ and $0.421 \mathrm{~km} \mathrm{~s}^{-1}$, respectively, as we mentioned in Section 2). Therefore, we looked for the emission features that consistently appear at the same position within uncertainty of a synthesized beam size and survive respectively in more than two and three consecutive velocity channels at $43 \mathrm{GHz}$ and $22 \mathrm{GHz}$, so that we could exclude spiky features with a very narrow line width, which are most likely artificial.

Then, we double checked the reliability of detections by cross-checking with Two Micron All Sky Survey (2MASS) images. Since the photosphere of RSGs exhibits a low effective temperature of about $3000 \mathrm{~K}$, a bright near IR counterpart with a red IR color should be found at the positions of maser sources if emission features are really originated in an RSG. We cross-checked between our radio images and 2MASS $K$-band images to find IR counterparts. As the angular resolution of 2MASS images is about 2".0 in $K$ band (Skrutskie et al. 2006), the resolution of the present observation seems to be sufficient enough for positional comparison. In considerations of beam sizes of both 2MASS and the present observations, we defined that emission is real if the 2MASS point sources with a red color are lying within 4 .'.5 and 2 ". 0 with respect to the radio positions of emission features at $43 \mathrm{GHz}$ and $22 \mathrm{GHz}$, respectively. Through these steps, finally we identified two real emission sources detected each in the $\mathrm{SiO}$ and $\mathrm{H}_{2} \mathrm{O}$ lines (the $\mathrm{SiO}$ and $\mathrm{H}_{2} \mathrm{O}$ lines are detected toward the same two sources). The details of the results are summarized in Section 3.2.

\subsection{Results of the $\mathrm{SiO}$ and $\mathrm{H}_{2} \mathrm{O}$ Maser Observations}

As mentioned in Section 3.1, we finally detected the $\mathrm{SiO}$ $v=1, J=1-0$ line toward two of four known RSGs (W 26 and $\mathrm{W} 237$ ) in $\mathrm{Wd} 1$, and also detected the $\mathrm{H}_{2} \mathrm{O} 6_{16}-5_{23}$ line toward the same two RSGs. In addition, we inspected across entire observing regions to doubly check further maser detections of unknown mass-losing evolved stars, but no other detections were found at a $3 \sigma$ level. In Table 3 we present the coordinate values of detected maser sources, peak and velocity-integrated intensities, intensity peak velocities, and rms noise levels. In Table 4, we present rms noise levels toward two known RSGs with no maser emission. In Figure 2, we present the line profiles of detected maser lines.

Both W 26 and W 237 exhibit asymmetric profiles (asymmetric with respect to the peak velocity) with a wide velocity range; the velocity ranges of W 26 and W 237 are roughly $40 \mathrm{~km} \mathrm{~s}^{-1}$ and $23 \mathrm{~km} \mathrm{~s}^{-1}$, respectively. Generally speaking, RSGs exhibit a 

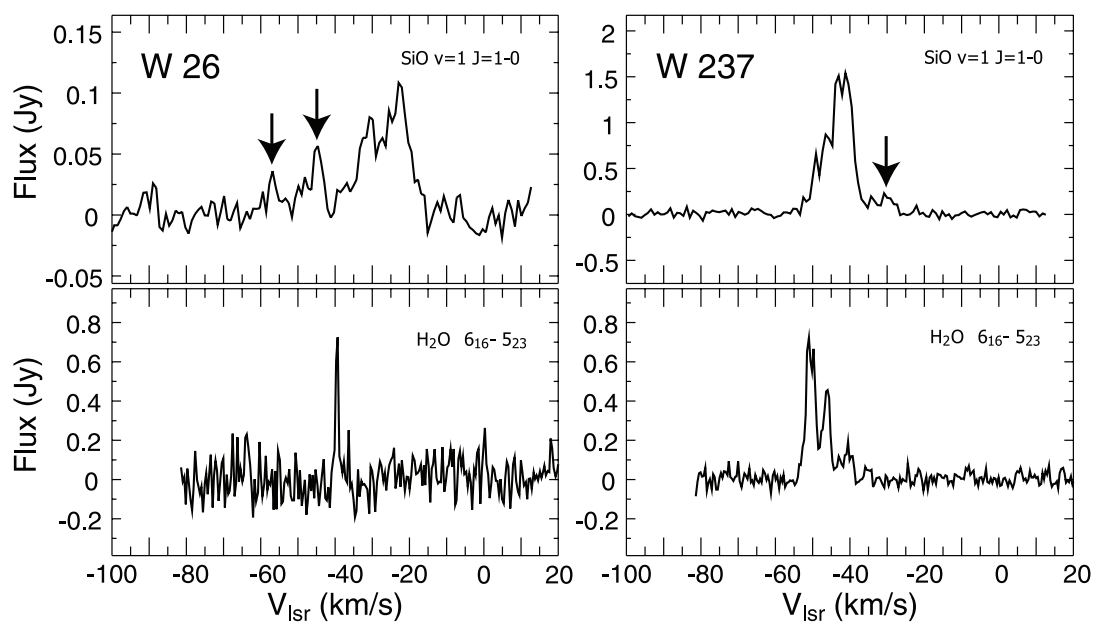

Figure 2. Spectra of the $\mathrm{SiO} v=1, J=1-0$ (upper panels) and $\mathrm{H}_{2} \mathrm{O} 6_{16}-5_{23}$ (lower panels) maser lines. The left and right panels show the line profiles of W 26 and $\mathrm{W} 237$, respectively. The arrows indicate weak components $(>5 \sigma)$ shifted from the primary peak (see the text).

Table 3

Parameters of Detected Maser Lines

\begin{tabular}{|c|c|c|c|c|c|c|}
\hline Source & $\begin{array}{c}\text { R.A. }^{\mathrm{a}} \\
(\mathrm{J} 2000.0)\end{array}$ & $\begin{array}{c}\text { Decl. }^{\mathrm{a}} \\
(\mathrm{J} 2000.0)\end{array}$ & $\begin{array}{c}I_{\text {peak }} \\
(\mathrm{Jy})\end{array}$ & $\begin{array}{c}I_{\text {int }} \\
\left(\mathrm{Jy} \mathrm{km} \mathrm{s}^{-1}\right)\end{array}$ & $\begin{array}{c}V_{\text {peak }} \\
\left(\mathrm{km} \mathrm{s}^{-1}\right)\end{array}$ & $\begin{array}{l}\text { rms } \\
(\mathrm{Jy})\end{array}$ \\
\hline \multicolumn{7}{|c|}{$\mathrm{SiO}(v=1, J=1-0)$} \\
\hline W 237 & $16: 47: 03.07$ & $-45: 52: 17.8$ & 1.54 & 16.82 & -41.2 & 0.027 \\
\hline W 26 & $16: 47: 05.15$ & $-45: 50: 35.5$ & 0.12 & 3.05 & -22.9 & 0.018 \\
\hline \multicolumn{7}{|c|}{$\mathrm{H}_{2} \mathrm{O}\left(6_{16}-5_{23}\right)$} \\
\hline W 237 & $16: 47: 03.12$ & $-45: 52: 19.2$ & 0.72 & 9.86 & -51.0 & 0.028 \\
\hline W 26 & $16: 47: 05.41$ & $-45: 50: 36.9$ & 0.73 & 3.29 & -39.3 & 0.094 \\
\hline
\end{tabular}

Note. ${ }^{a}$ Radio position obtained by fitting two-dimensional Gaussian function.

Table 4

Noise Levels Toward Non-detected RSGs

\begin{tabular}{lcc}
\hline \hline Source & $\begin{array}{c}\mathrm{rms}(\mathrm{SiO}) \\
(\mathrm{Jy})\end{array}$ & $\begin{array}{c}\mathrm{rms}\left(\mathrm{H}_{2} \mathrm{O}\right) \\
(\mathrm{Jy})\end{array}$ \\
\hline W 20 & 0.005 & 0.028 \\
W 75 & 0.009 & 0.094 \\
\hline
\end{tabular}

wider velocity range than that of asymptotic giant branch (AGB) stars, which exhibit a velocity range of, at most, $10 \mathrm{~km} \mathrm{~s}^{-1}$ in the $\mathrm{SiO}$ maser lines (see, e.g., Nakashima \& Deguchi 2007; Zhang et al. 2012a). Therefore, the velocity range of $\mathrm{SiO}$ maser emissions is consistent with the RSG status. Additionally, these two stars clearly exhibit asymmetric profiles (note that the black arrows in Figure 2 indicate weak component above a $5 \sigma$ level). This is also a typical characteristic often seen in RSGs (see, e.g., Nakashima \& Deguchi 2007; Zhang et al. 2012a). Both W 26 and W 237 exhibit clearly different intensities in the $\mathrm{SiO}$ line (the peak intensities of W 26 and W 237 are $0.12 \mathrm{Jy}$ and $1.54 \mathrm{Jy}$, respectively), while both stars exhibit almost the same intensity in the $\mathrm{H}_{2} \mathrm{O}$ line (the peak intensities of W 26 and W 237 are $0.73 \mathrm{Jy}$ and $0.72 \mathrm{Jy}$, respectively). Since the two stars are at the same distance in the same cluster, the difference in intensity directly reflects the difference of the absolute intensity of maser emission. This difference in intensity in the $\mathrm{SiO}$ maser line may be explained by the time variation in intensity of maser emission (see, e.g., Kamohara et al. 2005; Zhang et al. 2012a).

We shall note that the intensity of the $\mathrm{H}_{2} \mathrm{O}$ maser line is somewhat weaker than that of three well-known nearby (isolated) RSGs, i.e., VY CMa (1.2 kpc), VX Sgr (1.6 kpc), and NML Cyg $(1.6 \mathrm{kpc})$ exhibit the peak intensities of $199.8 \mathrm{Jy}$, $167.2 \mathrm{Jy}$, and $18.8 \mathrm{Jy}$ (these flux values are scaled at $3.5 \mathrm{kpc}$; Pashchenko et al. 2006; Choi et al. 2008; Zhang et al. 2012b), while W 237 and W 26 exhibit 0.72 Jy and 0.73 Jy. Here, we find roughly two order of differences in intensity between the two cases. Similarly, the $\mathrm{SiO}$ maser emission $(v=1, J=1-0)$ of the three well-known nearby RSGs exhibit systematically larger fluxes than RSGs in Wd1: VY CMa, VX Sgr, and NML Cyg exhibit the peak intensities of $5.4 \mathrm{Jy}, 31.4 \mathrm{Jy}$, and $2.6 \mathrm{Jy}$ (scaled at $3.5 \mathrm{kpc}$; Boboltz \& Claussen 2004; Zhang et al. 2012a; Su et al. 2012), while W 237 and W 26 exhibit $1.54 \mathrm{Jy}$ and $0.12 \mathrm{Jy}$, respectively. However, these differences in intensity between nearby RSGs and RSGs associated with Wd1 are partly due to the uncertainty of distances. In fact, the three representative nearby RSGs exhibit relatively large $12 \mu \mathrm{m}$ fluxes as well as the large maser intensities, i.e., the $12 \mu \mathrm{m}$ fluxes of VY CMa, VX Sgr, and NML Cyg are 1166 Jy (IRAS), 433 Jy (Midcourse Space Experiment, MSX), and $587 \mathrm{Jy}(M S X$; these flux values are scaled at $3.5 \mathrm{kpc}$ ), while those of W 237 and W 26 are $101 \mathrm{Jy}$, and $326 \mathrm{Jy}$, respectively. The systematically large midinfrared fluxes of the nearby RSGs means that the distances used for scaling may include a large uncertainty (even though a large dispersion in infrared intensities should be also taken into account). Although the distances to the nearby RSGs are based on the trigonometric parallax measurements giving a reliable distances, as we discuss later in Section 4 , the distance to Wd1 possibly includes a large uncertainty. Therefore, for more precise discussions on absolute maser intensities, we need more reliable distance information about $\mathrm{Wd} 1$. 
On the other hand, the intensity ratios of the $\mathrm{H}_{2} \mathrm{O}$ to $\mathrm{SiO}$ maser intensities are distance-free parameters, and the nearby RSGs seem to exhibit a relatively large value on average: the peak intensity ratios of the $\mathrm{H}_{2} \mathrm{O}$ to $\mathrm{SiO}$ maser lines of VY CMa, VX Sgr, and NML Cyg are 36.9, 5.3, and 7.3, while those of W 237 and W 26 are 0.47 and 6.1. Even though the result is not very obvious (because only VY CMa and W 237 exhibit extreme values), one may consider that this relatively weak intensity of the $\mathrm{SiO}$ maser line of RSGs in Wd1 is somehow originated in the cluster environment; for example, the outer layer of the envelope may be disturbed by interactions with ambient materials in the condensed cluster environment.

It is notable that $\mathrm{SiO}$ maser emission of W 26 and W 237 exhibits larger velocity ranges than $\mathrm{H}_{2} \mathrm{O}$ emission. In the case of spherical envelopes, the velocity range of $\mathrm{SiO}$ emission theoretically should be smaller than that of $\mathrm{H}_{2} \mathrm{O}$ emission, because molecular gas is usually still being accelerated in the radial direction in the innermost region $\left(2-3 R_{*}\right)$, from which $\mathrm{SiO}$ maser is emitted. Since maser emission cannot be amplified in the direction of acceleration, $\mathrm{SiO}$ masers are usually amplified only in the tangential direction to the radial direction. Therefore, in the case of a spherical envelope, intensity peaks of $\mathrm{SiO}$ maser emission are found at velocities close to the systemic velocity. On the contrary, at the $\mathrm{H}_{2} \mathrm{O}$ maser region (20-30 $R_{*}$ ) the motion of molecular gases usually already reaches to almost the terminal velocity and, in addition, gas is usually turbulent. Therefore, the maser can be amplified in any direction. As a result, the velocity range of $\mathrm{H}_{2} \mathrm{O}$ maser emission is generally wider than that of the $\mathrm{SiO}$ maser emission. The present result possibly suggests that circumstellar envelopes of observed RSGs somewhat deviate from spherical symmetry. We shall note, however, that this discussion may be affected by the time variation of the line profile and sensitivity of the observation if weak components exist below the detection limit (for example, as mentioned later in Section 4, the convective motion could alter the line profile).

\section{SYSTEMIC VELOCITIES OF MASER SOURCES}

In the case of AGB stars, it is well known that the peak velocity of $\mathrm{SiO}$ maser emission represents the stellar systemic velocity within an uncertainty of $2-3 \mathrm{~km} \mathrm{~s}^{-1}$ (Jewell et al. 1984). In the case of RSGs, however, the peak intensity clearly does not represent the systemic velocity of the stars, because the line profiles are not symmetric in velocity. For that reason, here we estimate the systemic velocities of RSGs in Wd1 by assuming that the median velocity of a line profile is close to the stellar systemic velocity: specifically, we calculated the average of the highest and lowest velocities of $5 \sigma$ channels. In the case of $\mathrm{SiO}$ maser emission, the derived values of the median velocities for $\mathrm{W} 26$ and $\mathrm{W} 237$ are $-38.1 \mathrm{~km} \mathrm{~s}^{-1}$ and $-41.7 \mathrm{~km} \mathrm{~s}^{-1}$, respectively. Similarly, the median velocities obtained from the $\mathrm{H}_{2} \mathrm{O}$ maser profiles are $-39.3 \mathrm{~km} \mathrm{~s}^{-1}$ and $-48.5 \mathrm{~km} \mathrm{~s}^{-1}$ for W 26 and W 237, respectively (since W 26 exhibits a singlepeak profile in the $\mathrm{H}_{2} \mathrm{O}$ line, here we simply give the peak velocity). These derived values are not exactly consistent with the systemic velocities obtained by infrared high-dispersion spectroscopy of the CO band head at $2.29 \mu \mathrm{m}$ (near-infrared velocities are $-44.5 \mathrm{~km} \mathrm{~s}^{-1}$ and $-57.5 \mathrm{~km} \mathrm{~s}^{-1}$ for $\mathrm{W} 26$ and W 237, respectively; Mengel \& Tacconi-Garman 2009). The differences between the present observation and near-infrared measurement are roughly $5-6 \mathrm{~km} \mathrm{~s}^{-1}$ and $9-16 \mathrm{~km} \mathrm{~s}^{-1}$ for W 26 and $\mathrm{W} 237$, respectively.

As we mentioned in Section 2, we checked the reliability of our velocity calibration by observing a known maser source with
Table 5

Summary of Velocity Measurements of Westerlund 1

\begin{tabular}{lcc}
\hline \hline Measurement & $\begin{array}{c}\text { Averaged Velocity } \\
\left(\mathrm{km} \mathrm{s}^{-1}\right)\end{array}$ & Reference \\
\hline $\mathrm{SiO}$ maser & -40 & See Section 4 \\
$\mathrm{H}_{2} \mathrm{O}$ maser & -44 & See Section 4 \\
$\mathrm{CO}$ band head & -51 & Mengel \& Tacconi-Garman (2009) \\
$\mathrm{CO}$ emission & $-48 \pm 14$ & Luna et al. (2009) \\
H I absorption & $-55_{-26}^{+9}$ & Kothes \& Dougherty (2007) \\
\hline
\end{tabular}

a known systemic velocity, and confirmed that the present velocity measurement is consistent with a previous measurement (Haikala et al. 1994). On the other hand, the resolution of near-IR observations (Mengel \& Tacconi-Garman 2009) was also pretty high $\left(R \sim 9000\right.$; this corresponds to $\left.\Delta v=33 \mathrm{~km} \mathrm{~s}^{-1}\right)$, and the accuracy of the infrared velocity measurement must reach to, at least, a several $\mathrm{km} \mathrm{s}^{-1}$ level, because a series of absorption lines were simultaneously observed and the derived velocities were averaged out. Therefore, inconsistency in the velocities seems to be originated in astrophysical reasons. In fact, the atmosphere of RSGs are strongly affected by convective motions: the maximum expansion velocities of RSG envelopes could be often beyond 30-40 $\mathrm{km} \mathrm{s}^{-1}$ due to convective motions (Josselin $\&$ Plez 2005). In addition, the infrared velocity determined by observing the $\mathrm{CO}$ band head may be affected by the pulsation of RSGs; for example, in the case of M-type miras, the pulsation is known to shift the position of the CO band head up to $15 \mathrm{~km} \mathrm{~s}^{-1}$ (Scholz \& Wood 2000). Presumably, the difference in velocities up to $16 \mathrm{~km} \mathrm{~s}^{-1}$ may be interpreted for these reasons.

The average of two systemic velocities of $\mathrm{SiO}$ maser emission is calculated to be $-39.9 \mathrm{~km} \mathrm{~s}^{-1}$. Similarly, in the case of $\mathrm{H}_{2} \mathrm{O}$ maser emission, the average is $-43.9 \mathrm{~km} \mathrm{~s}^{-1}$. These values are expected to be close to the systemic velocity of Wd1. Previously, two indirect measurements of the systemic velocity of $\mathrm{Wd} 1$ have been made based on the motion of ambient interstellar gas ( $\mathrm{H} \mathrm{I}$ and $\mathrm{CO}$ ) surrounding Wd1: $-55_{-26}^{+9} \mathrm{~km} \mathrm{~s}^{-1}$ (H I; Kothes \& Dougherty 2007) and $-48 \pm 14 \mathrm{~km} \mathrm{~s}^{-1}$ (CO; Luna et al. 2009). The present values are not inconsistent with these values if we consider the uncertainty (the systemic velocities of $\mathrm{Wd} 1$ are briefly summarized in Table 5).

Using the systemic velocity derived, we calculated the kinematic distance to Wd1 under the assumption of a flat circular rotation model of the Galaxy (here, we use the velocity of $\mathrm{SiO}$ maser emission as a representative value): in the calculation we followed the same methodology adopted by Kothes \& Dougherty (2007), assuming the galactocentric distance of $7.6 \mathrm{kpc}$, and the derived kinematic distance is $3.0 \pm 0.5 \mathrm{kpc}$ (here, we assume an uncertainty of $10 \mathrm{~km} \mathrm{~s}^{-1}$ in the velocity of $\mathrm{Wd} 1$ based on the above discussion). We also calculated the kinematic distance based on Reid et al. (2009) for comparison, and found no significant differences from those based on Kothes \& Dougherty (2007): the obtained kinematic distances are summarized in Table 6. On the other hand, the obtained kinematic distance is slightly nearer than luminosity distances derived by independent methodologies using main-sequence stars, OB supergiants, and Wolf-Rayet stars in Wd1 (3.5-5.5 kpc; Crowther et al. 2006; Brandner et al. 2008; Negueruela et al. 2010a). Therefore, in the following analysis, we adopt $3.5 \mathrm{kpc}$ as the representative distance to $\mathrm{Wd} 1$, because this distance is not inconsistent with both the kinematic and luminosity distances (at the distance of $3.5 \mathrm{kpc}$, absolute luminosities of W 26 and W 237 
Table 6

Parameters of Clusters

\begin{tabular}{lccccccc}
\hline \hline Cluster & Adopted Distance & Galactocentric Distance & Averaged $V_{\mathrm{lsr}}$ & Reference & $\begin{array}{c}\text { Kin. Distance } \\
\text { kot07 } 7^{\mathrm{a}} \\
(\mathrm{kpc})\end{array}$ & $\begin{array}{c}\text { Kin. Distance } \\
\text { rei09 } \\
(\mathrm{kpc})\end{array}$ & $\begin{array}{c}\text { Averaged A } \\
(\mathrm{mag})\end{array}$ \\
\hline Wd1 & $(\mathrm{kpc})$ & $(\mathrm{kpc})$ & $\left(\mathrm{km} \mathrm{s}^{-1}\right)$ & & 3.0 & 3.0 & 1.33 \\
RSGC1 & 3.5 & 4.5 & -39.9 & 1 & 6.9 & 6.0 & 2.57 \\
RSGC2 & 6.6 & 3.3 & 120.9 & 2 & 3.2 & 5.1 & 1.40 \\
RSGC2 SW & 5.5 & 3.6 & 96.6 & 3 & 5.2 & 5.2 & 2.52 \\
Per OB1 & 5.5 & 3.6 & -38.5 & 4 & 3.3 & 3.0 & 0.31 \\
Mc8 & 2.4 & 9.5 & 95.0 & 3 & 5.2 & 5.2 & 1.11 \\
\hline
\end{tabular}

Notes.

${ }^{\text {a }}$ Calculated based on the Galaxy model of Kothes \& Dougherty (2007).

${ }^{\mathrm{b}}$ Calculated based on the Galaxy model of Reid et al. (2009).

References. ${ }^{1}$ see Section $4 ;{ }^{2}$ Davies et al. (2008); ${ }^{3}$ Deguchi et al. (2010); ${ }^{4}$ Asaki et al. (2010).

are respectively calculated to be $1.1 \times 10^{6} L_{\odot}$ and $2.3 \times 10^{5} L_{\odot}$ based on the photometric data mentioned in Section 5.1; these values are roughly consistent with a range of typical absolute luminosities of RSGs $\left(2 \times 10^{4} L_{\odot}-6 \times 10^{6} L_{\odot}\right.$, see, e.g., Stothers $\&$ Chin 1999; Mauron \& Josselin 2011)).

\section{DISCUSSION}

Our primary purpose of this project is to explore possibilities of using maser emission for investigating massive clusters and associated RSGs. We previously discussed the kinematics of the cluster itself using the velocity information of RSGs with maser emission (Nakashima \& Deguchi 2006). However, since the number of detection in the present observation is not sufficient enough for discussing cluster kinematics, here we focus on the nature of RSGs. In the following subsections, first we compare infrared properties of four known RSGs to clarify the difference and similarity between detections and non-detections in the $\mathrm{SiO}$ and $\mathrm{H}_{2} \mathrm{O}$ maser observations. Second, we extend our analysis to RSGs in other massive clusters, which previously have been observed in the $\mathrm{SiO}, \mathrm{H}_{2} \mathrm{O}$, and $\mathrm{OH}$ maser lines, so that we can more comprehensively consider the characteristics of RSGs with and without maser emission.

\subsection{Comparison between Detections and Non-detections}

The infrared data used for the present analysis were taken from following data archives: Deep Near Infrared Survey of the Southern Sky (DENIS; Deul et al. 1995), 2MASS Point Source Catalog (PSC; Skrutskie et al. 2006), Spitzer's Galactic Legacy Infrared Mid-Plane Survey Extraordinaire (GLIMPSE; Werner et al. 2004), MSX (Egan et al. 2003), Wide-field Infrared Survey Explorer (WISE) All Sky Source Catalog (Wright et al. 2010), and AKARI/IRC Point Source Catalog (Ishihara et al. 2010). In conjunction with these archival data, we obtained 8-18 photometric data points per each RSG from $0.82 \mu \mathrm{m}$ to $22 \mu \mathrm{m}$. The photometric data collected are given in Appendix B.

It has been known that interstellar extinction toward $\mathrm{Wd} 1$ is non-negligible in near-infrared wavelengths. Therefore, we adopted an extinction correction for photometric data below $10 \mu \mathrm{m}$ in the following way. First, we found $E_{J-K}$ values from Levesque et al. (2005) and Mengel \& Tacconi-Garman (2007) toward each RSG; their $E_{J-K}$ values are based on intrinsic $J-K$ colors determined by effective temperatures of theoretical models (Levesque et al. 2005; Mengel \& Tacconi-Garman 2007). Second, $A_{K}$ values toward each RSG are determined using the formula given in Crowther et al. (2006) (note that some authors recently use a slightly different formula (see, e.g., Nishiyama et al. 2006), but the difference of the formulae does not affect the results of our discussion; see, some more details in Section 5.2). Finally, derived extinction at $K$ band is extrapolated to other wavelengths using the formula given in Indebetouw et al. (2005). The averaged extinction toward four known RSGs at $K$ band is 1.33. This averaged extinction is consistent with previous studies; for example, the extinction measurements using main-sequence and pre-main-sequence stars in Wd1 show $A_{K}$ is roughly 1.13 (Brandner et al. 2008), the result of Wolf-Rayet stars shows $A_{K}$ is 1.01 (Crowther et al. 2008), and the results of OB supergiants are 1.20 (Clark et al. 2005) and 1.34 (Negueruela et al. 2010a). Both original and interstellar extinction corrected spectral energy distribution (SED) diagrams are given in Figure 3.

To estimate physical parameters of dust envelopes, SED profiles were fitted by one-dimensional dust radiative transfer models assuming centrally heated spherical density distributions using the DUSTY code (Ivezić et al. 1999). The central star (i.e., central heat source) was assumed to be a point source at the center of the SEDs and their SEDs were taken to be Planckian. We used the grain type of cold (Sil-Oc) silicates (Ossenkopf et al. 1992). The standard Mathis, Rumpl, Nordsieck (Mathis et al. 1977) power law was used for the grain size distributions. The dust temperature on the inner shell boundary and the optical depth were varied assuming an inverse square law for the spherical density distribution. The shell was assumed to extend to 10,000 times its inner radius. In order to model the dust shells of RSGs, we surveyed the numerical space of three parameters, i.e., the effective temperature of the central heating source ( $\left.T_{\text {eff }}\right)$, dust temperature on the inner shell boundary $\left(T_{\mathrm{d}}\right)$, and optical depth at $2.2 \mu \mathrm{m}\left(\tau_{2.2}\right)$. The ranges and steps of parameter searches were $1500-4000 \mathrm{~K}$ and $100 \mathrm{~K}, 100-1800 \mathrm{~K}$ and $100 \mathrm{~K}$, and $0.001-0.05$ and 0.001 for $T_{\text {eff }}, T_{\mathrm{d}}$, and $\tau_{2.2}$, respectively. Additionally, we inspected a couple of models with large optical depths $\left(\tau_{2.2}>0.05\right)$, but we confirmed, in such a range of large optical depths, that there are no chances to fit the observational SEDs due to the strong absorption of silicate at $9.7 \mu \mathrm{m}$. We adopted the fits for which the sum of squares of the deviations between the observed and modeled fluxes (after scaling) were minimum. The mass-loss rate calculated by DUSTY posses general scaling properties in terms of the luminosity $L$, gas-todust mass ratio $r_{\mathrm{gd}}$, and dust grain bulk density $\rho_{\mathrm{s}}$, assuming $L=10^{4} L_{\odot}, r_{\mathrm{gd}}=200$, and $\rho_{\mathrm{s}}=3 \mathrm{~g} \mathrm{~cm}^{-3}$. The mass-loss rate is scaled in proportion to $L^{3 / 4}\left(r_{\mathrm{gd}} \rho_{\mathrm{S}}\right)^{1 / 2}$ (this quantity has roughly 30\% inherent uncertainty; Ivezić et al. 1999). To obtain 

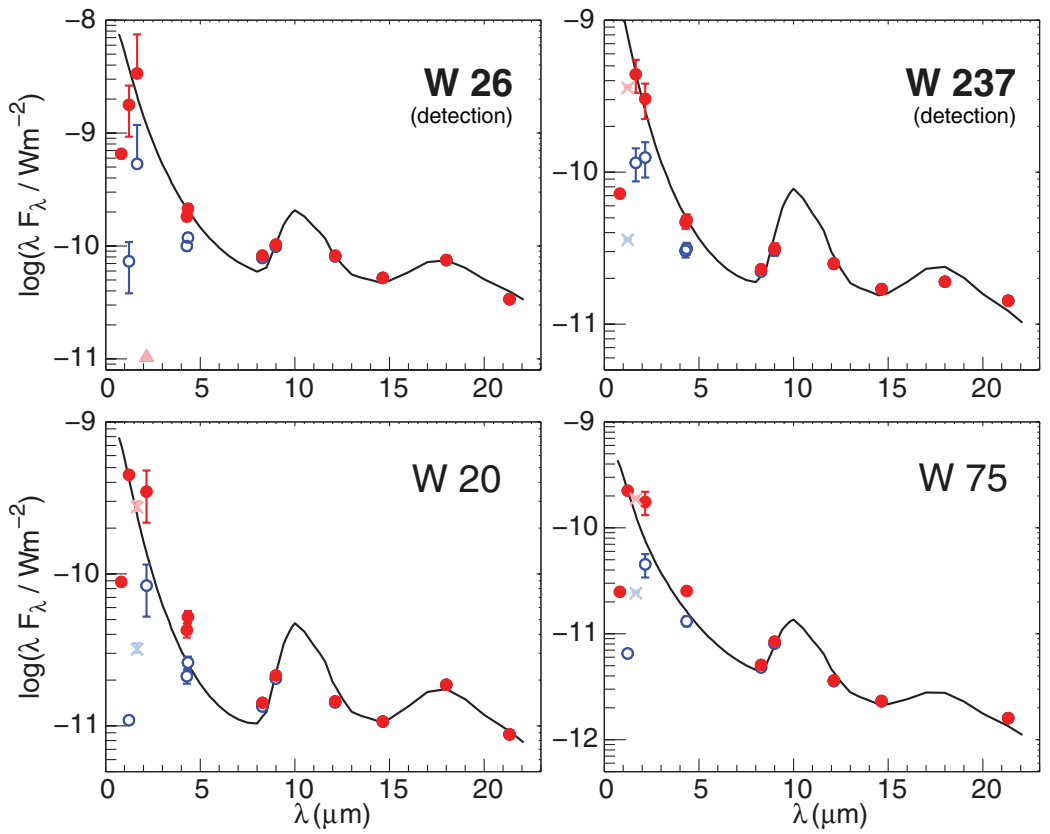

Figure 3. Spectral energy distributions of four known RSGs in Wd1. The blue open circles are data points of original values without interstellar extinction corrections, and the red filled circles are data points with interstellar extinction corrections. The light-blue and light-red triangles represent the lower limits of fluxes, respectively, before and after adopting interstellar extinction correction, and the light-blue and light-red crosses represent the flux values with a low quality flag, respectively, before and after adopting interstellar extinction correction. The black curves are the results of model fitting with the DUSTY code (see the text for details).

(A color version of this figure is available in the online journal.)

Table 7

DUSTY Model Parameters and Absolute Luminosities

\begin{tabular}{lccccc}
\hline \hline $\begin{array}{l}\text { Source } \\
\text { Name }\end{array}$ & $\begin{array}{c}T_{\text {eff }} \\
(\mathrm{K})\end{array}$ & $\begin{array}{c}T_{d} \\
(\mathrm{~K})\end{array}$ & $\tau_{2.2}$ & $\begin{array}{c}\log \dot{M} \\
\left(M_{\odot} \mathrm{yr}^{-1}\right)\end{array}$ & $\begin{array}{r}\log L \\
\left(L_{\odot}\right)\end{array}$ \\
\hline W 237 & 3600 & 600 & 0.004 & -5.00 & 5.37 \\
W 26 & 3700 & 500 & 0.002 & -4.65 & 6.03 \\
W 20 & 3500 & 500 & 0.005 & -5.07 & 5.10 \\
W 75 & 3600 & 1100 & 0.004 & -5.69 & 4.83 \\
\hline
\end{tabular}

the mass-loss rate of each RSG in Wd1, first we calculated the absolute luminosity of each RSG: we integrated flux densities by approximating the area under SED profiles by trapezoids, and the absolute luminosity was derived by assuming the distance of $3.5 \mathrm{kpc}$. The derived absolute luminosities $(\log L)$ are 5.37, $6.03,5.10$, and 4.83 for $\mathrm{W} 237, \mathrm{~W} 26, \mathrm{~W} 20$, and $\mathrm{W} 75$, respectively. Then, using these absolute luminosities, we scaled the mass-loss rate, assuming $r_{\mathrm{gd}}=200$ and $\rho_{\mathrm{s}}=3 \mathrm{~g} \mathrm{~cm}^{-3}$. Consequently, the mass-loss rates $(\log \dot{M})$ are calculated to be $-5.00,-4.65,-5.07$, and -5.69 for $\mathrm{W} 237, \mathrm{~W} 26$, W 20, and W 75, respectively. The best-fit input parameters of the DUSTY modeling obtained mass-loss rates and absolute luminosities used for scaling are summarized in Table 7.

Even though the derived mass-loss rates and luminosities are within a typical range of the mass-loss rate of RSGs (Mauron \& Josselin 2011), W 75, which is a non-detection in both the $\mathrm{SiO}$ and $\mathrm{H}_{2} \mathrm{O}$ maser lines, exhibits the smallest luminosity and massloss rate, while two detections (W 237 and W 26) clearly exhibit larger absolute luminosities and mass-loss rates than $\mathrm{W} 75$. According to the results of the above analysis, one may expect that RSGs with maser emission are found predominantly in RSGs with relatively large mass-loss rates and absolute luminosities.

\subsection{Consideration Using Previous Maser Observations}

As discussed in Section 5.1, the detection rates of maser emission of RSGs seem to be related to infrared properties.
However, the number of RSGs in Wd1 is unfortunately not sufficient to form a conclusion. Therefore, in this section, we extend our analysis to RSGs associated with other clusters (i.e., RSGC1, RSGC2, RSGC2 SW, Per OB1, Mc8). Since the galactocentric distance of these five clusters is, more or less, similar (except for Per OB1; see Table 6), we can expect that the effects of metallicity gradient in the galactic disk (Henry \& Worthey 1999) are minimized. Unfortunately, at this moment, the distance information is based on radial velocities (i.e., distances are estimated by assuming the flat rotation model of the Galaxy). Therefore, the distances could include a relatively large uncertainty (relative error of more than 50\% according to the recent results of trigonometric parallax measurements; H. Imai 2012, private communication; theoretically, however, the distances to massive clusters may be improved in the future, because there are independent methodologies to measure the distances to clusters (see, e.g., Perryman et al. 1998; An et al. 2007)). Here, as a preliminary analysis, we discuss based on the kinematic distances estimated from the radial velocities.

In the five massive clusters mentioned above, in total 53 RSGs have been identified until now (Humphreys 1970; Figer et al. 2006; Davies et al. 2007; Deguchi et al. 2010). As we did in Section 5.1, we collected infrared photometric data of RSGs from DENIS, 2MASS PSC, Spitzer/GLIMPSE, MSX, WISE All Sky Source Catalog, and AKARI/IRC PSC. The collected infrared photometric data and SED plots are summarized again 
Table 8

DUSTY Model Parameters and Absolute Luminosities

\begin{tabular}{|c|c|c|c|c|c|}
\hline $\begin{array}{l}\text { Source } \\
\text { Name }\end{array}$ & $\begin{array}{l}T_{\text {eff }} \\
(\mathrm{K})\end{array}$ & $\begin{array}{c}T_{d} \\
(\mathrm{~K})\end{array}$ & $\tau_{2.2}$ & $\begin{array}{c}\log \dot{M} \\
\left(M_{\odot} \mathrm{yr}^{-1}\right)\end{array}$ & $\begin{array}{l}\log L \\
\left(L_{\odot}\right)\end{array}$ \\
\hline \multicolumn{6}{|c|}{ RSGC1 } \\
\hline F01 & 3300 & 300 & 0.005 & -4.65 & 5.41 \\
\hline F02 & 3600 & 300 & 0.004 & -4.58 & 5.56 \\
\hline F03 & 3200 & 400 & 0.002 & -5.24 & 5.22 \\
\hline F04 & 3900 & 500 & 0.001 & -5.46 & 5.24 \\
\hline F05 & 3600 & 400 & 0.001 & -5.42 & 5.22 \\
\hline F06 & 3300 & 400 & 0.001 & -5.61 & 5.01 \\
\hline F07 & 3600 & 1800 & 0.001 & -6.36 & 4.89 \\
\hline F08 & 3700 & 300 & 0.001 & -5.22 & 5.30 \\
\hline F09 & 3100 & 500 & 0.003 & -5.30 & 5.10 \\
\hline F10 & 3500 & 200 & 0.001 & -5.20 & 5.09 \\
\hline F11 & 3600 & 1200 & 0.002 & -5.73 & 5.14 \\
\hline F12 & 3200 & 1000 & 0.001 & -6.04 & 4.98 \\
\hline F13 & 4100 & 500 & 0.001 & -5.32 & 5.40 \\
\hline F14 & 3500 & 1500 & 0.001 & -6.44 & 4.67 \\
\hline \multicolumn{6}{|c|}{ RSGC2 } \\
\hline St2-01 & 3800 & 1400 & 0.003 & -6.06 & 4.59 \\
\hline St2-02 & 3500 & 1500 & 0.001 & -6.47 & 4.62 \\
\hline St2-03 & 3700 & 900 & 0.003 & -5.39 & 5.20 \\
\hline St2-04 & 3700 & 800 & 0.001 & -5.91 & 4.93 \\
\hline St2-05 & 3400 & 1200 & 0.001 & -6.33 & 4.67 \\
\hline \multicolumn{6}{|c|}{ RSGC2 SW } \\
\hline$\overline{\text { St2-08 }}$ & 3300 & 600 & 0.001 & -6.12 & 4.55 \\
\hline St2-11 & 3700 & 600 & 0.002 & -5.41 & 5.12 \\
\hline St2-14 & 3500 & 800 & 0.001 & -5.90 & 4.96 \\
\hline St2-18 & 3200 & 500 & 0.003 & -4.87 & 5.64 \\
\hline St2-26 & 3300 & 500 & 0.001 & -6.14 & 4.42 \\
\hline \multicolumn{6}{|c|}{ Per OB1 } \\
\hline W Per & 3400 & 600 & 0.004 & -5.24 & 5.09 \\
\hline S Per & 3600 & 900 & 0.010 & -4.95 & 5.27 \\
\hline T Per & 3500 & 1100 & 0.001 & -6.31 & 4.62 \\
\hline V605 Cas & 3600 & 500 & 0.001 & -6.00 & 4.56 \\
\hline V778 Cas & 3500 & 1200 & 0.001 & -6.36 & 4.61 \\
\hline PR Per & 3300 & 500 & 0.001 & -6.01 & 4.59 \\
\hline FZ Per & 3400 & 1300 & 0.003 & -6.15 & 4.48 \\
\hline $\mathrm{BD}+59372$ & 3100 & 1500 & 0.001 & -6.93 & 4.08 \\
\hline XX Per & 3700 & 900 & 0.002 & -5.73 & 4.93 \\
\hline HD 236947 & 3400 & 1500 & 0.001 & -6.69 & 4.35 \\
\hline KK Per & 3500 & 1300 & 0.002 & -6.00 & 4.85 \\
\hline V550 Per & 3500 & 1500 & 0.002 & -6.51 & 4.27 \\
\hline PP Per & 3700 & 1500 & 0.001 & -6.67 & 4.32 \\
\hline BU Per & 3300 & 400 & 0.002 & -5.51 & 4.83 \\
\hline AD Per & 3400 & 900 & 0.001 & -6.01 & 4.90 \\
\hline SU Per & 3700 & 700 & 0.001 & -5.72 & 5.10 \\
\hline RS Per & 3500 & 900 & 0.003 & -5.53 & 5.04 \\
\hline V439 Per & 3600 & 1500 & 0.001 & -6.52 & 4.54 \\
\hline V403 Per & 3500 & 1500 & 0.001 & -6.70 & 4.31 \\
\hline V441 Per & 3200 & 1000 & 0.002 & -5.95 & 4.78 \\
\hline YZ Per & 3400 & 800 & 0.002 & -5.64 & 4.84 \\
\hline GP Cas & 3300 & 800 & 0.002 & -5.84 & 4.78 \\
\hline V648 Cas & 3700 & 700 & 0.001 & -5.79 & 5.01 \\
\hline \multicolumn{6}{|c|}{ Mc8 } \\
\hline Mc8-01 & 3200 & 600 & 0.004 & -5.66 & 4.56 \\
\hline Mc8-02 & 3200 & 600 & 0.001 & -6.27 & 4.37 \\
\hline Mc8-03 & 3300 & 900 & 0.010 & -5.85 & 4.12 \\
\hline Mc8-04 & 3300 & 1400 & 0.015 & -5.77 & 4.35 \\
\hline Mc8-05 & 3200 & 900 & 0.002 & -6.18 & 4.41 \\
\hline Mc8-06 & 3100 & 1500 & 0.003 & -6.16 & 4.62 \\
\hline
\end{tabular}


Table 9

Summary of Previous Maser Observations of Other Clusters 1

\begin{tabular}{|c|c|c|c|c|c|c|}
\hline \multirow{2}{*}{$\begin{array}{l}\text { Source } \\
\text { Name }\end{array}$} & \multirow{2}{*}{$\begin{array}{l}\text { IRAS } \\
\text { Name }\end{array}$} & \multicolumn{2}{|c|}{ Coordinate } & \multicolumn{3}{|c|}{ Maser Observation } \\
\hline & & $\begin{array}{c}\text { R.A. } \\
\text { (J 2000.0) }\end{array}$ & $\begin{array}{c}\text { Decl. } \\
\text { (J 2000.0) }\end{array}$ & $\mathrm{SiO}$ & $\mathrm{H}_{2} \mathrm{O}$ & $\mathrm{OH}$ \\
\hline \multicolumn{7}{|c|}{ RSGC1 } \\
\hline F01 & $\ldots$ & $18: 37: 56.30$ & $-06: 52: 32.2$ & $y^{1}$ & $\mathrm{n}^{1}$ & $\mathrm{n}^{2}$ \\
\hline F02 & $\ldots$ & $18: 37: 55.28$ & $-06: 52: 48.3$ & $\mathrm{y}^{1}$ & $\mathrm{n}^{1}$ & $n^{2}$ \\
\hline F03 & $\ldots$ & $18: 37: 59.73$ & $-06: 53: 49.2$ & $\mathrm{n}^{1}$ & $\mathrm{n}^{1}$ & $n^{2}$ \\
\hline F04 & $\ldots$ & $18: 37: 50.88$ & $-06: 53: 38.1$ & $y^{1}$ & $\mathrm{n}^{1}$ & $\mathrm{n}^{2}$ \\
\hline F05 & $\ldots$ & $18: 37: 55.52$ & $-06: 52: 11.6$ & $\mathrm{n}^{1}$ & $\mathrm{n}^{1}$ & $n^{2}$ \\
\hline F06 & $\ldots$ & $18: 37: 57.45$ & $-06: 53: 25.3$ & $\mathrm{n}^{1}$ & $\mathrm{n}^{1}$ & $n^{2}$ \\
\hline F07 & $\ldots$ & $18: 37: 54.31$ & $-06: 52: 34.5$ & $\mathrm{n}^{1}$ & $\mathrm{n}^{1}$ & $n^{2}$ \\
\hline F08 & $\ldots$ & $18: 37: 55.19$ & $-06: 52: 10.7$ & $\ldots$ & $\ldots$ & $n^{2}$ \\
\hline F09 & $\ldots$ & $18: 37: 57.76$ & $-06: 52: 22.1$ & $\mathrm{n}^{1}$ & $\mathrm{n}^{1}$ & $n^{2}$ \\
\hline F10 & $\ldots$ & $18: 37: 59.53$ & $-06: 53: 31.9$ & $\mathrm{n}^{1}$ & $\mathrm{n}^{1}$ & $n^{2}$ \\
\hline F11 & $\ldots$ & $18: 37: 51.72$ & $-06: 51: 49.7$ & $\mathrm{n}^{1}$ & $\mathrm{n}^{1}$ & $n^{2}$ \\
\hline F12 & $\ldots$ & $18: 38: 03.30$ & $-06: 52: 45.1$ & $\mathrm{n}^{1}$ & $\mathrm{n}^{1}$ & $n^{2}$ \\
\hline $\mathrm{F} 13$ & $\ldots$ & $18: 37: 58.91$ & $-06: 52: 32.1$ & $y^{1}$ & $y^{1}$ & $y^{2}$ \\
\hline F14 & $\ldots$ & $18: 37: 47.65$ & $-06: 53: 02.1$ & $\mathrm{n}^{1}$ & $\mathrm{n}^{1}$ & $\mathrm{n}^{2}$ \\
\hline \multicolumn{7}{|c|}{ RSGC2 } \\
\hline St2-01 & $\ldots$ & $18: 39: 19.88$ & $-06: 01: 48.0$ & $\mathrm{n}^{3}$ & $\mathrm{n}^{3}$ & $\ldots$ \\
\hline St2-02 & $\ldots$ & $18: 39: 18.25$ & $-06: 02: 14.2$ & $n^{3}$ & $\mathrm{n}^{3}$ & $\ldots$ \\
\hline St2-03 & IRAS $18366-0603$ & $18: 39: 19.59$ & $-06: 00: 41.6$ & $y^{3}$ & $y^{3}$ & $\mathrm{n}^{4}$ \\
\hline St2-04 & $\ldots$ & $18: 39: 24.60$ & $-06: 02: 13.9$ & $n^{3}$ & $n^{3}$ & $\ldots$ \\
\hline St2-05 & $\ldots$ & $18: 39: 14.70$ & $-06: 01: 36.5$ & $n^{3}$ & $n^{3}$ & $\ldots$ \\
\hline \multicolumn{7}{|c|}{ RSGC2 SW } \\
\hline St2-08 & $\ldots$ & 18:39:07.75 & $-06: 03: 20.2$ & $\mathrm{n}^{3}$ & $y^{3}$ & $\ldots$ \\
\hline St2-11 & $\ldots$ & 18:39:05.59 & $-06: 04: 26.6$ & $y^{3}$ & $y^{3}$ & $\ldots$ \\
\hline St2-14 & $\ldots$ & 18:39:08.04 & $-06: 05: 24.2$ & $n^{3}$ & $n^{3}$ & $\ldots$ \\
\hline St2-18 & $\ldots$ & $18: 39: 02.38$ & $-06: 05: 10.6$ & $y^{3}$ & $y^{3}$ & $\cdots$ \\
\hline St2-26 & $\ldots$ & $18: 38: 56.98$ & $-06: 06: 45.7$ & $y^{3}$ & $\mathrm{n}^{3}$ & $\ldots$ \\
\hline \multicolumn{7}{|c|}{ Per OB1 } \\
\hline W Per & IRAS $02469+5646$ & $02: 50: 37.91$ & $56: 59: 00.1$ & $y^{5}$ & $\mathrm{n}^{6}$ & $\ldots$ \\
\hline S Per & IRAS $02192+5821$ & $02: 22: 51.73$ & $58: 35: 11.2$ & $y^{7}$ & $y^{8}$ & $y^{9}$ \\
\hline T Per & IRAS $02157+5843$ & $02: 19: 21.87$ & $58: 57: 40.3$ & $\mathrm{n}^{10}$ & $\ldots$ & $\ldots$ \\
\hline V605 Cas & IRAS $02167+5926$ & $02: 20: 22.48$ & $59: 40: 16.8$ & $\mathrm{n}^{10}$ & $\ldots$ & $\ldots$ \\
\hline V778 Cas & IRAS $01550+5901$ & 01:58:28.90 & $59: 16: 08.7$ & $\mathrm{n}^{10}$ & $\ldots$ & $\ldots$ \\
\hline PR Per & IRAS $02181+5738$ & $02: 21: 42.41$ & $57: 51: 46.0$ & $\mathrm{n}^{10}$ & $\ldots$ & $\ldots$ \\
\hline FZ Per & IRAS $02174+5655$ & $02: 20: 59.65$ & $57: 09: 30.0$ & $\mathrm{n}^{10}$ & $\ldots$ & $\ldots$ \\
\hline BD +59 372 & IRAS $01561+6000$ & 01:59:39.67 & $60: 15: 01.9$ & $\ldots$ & $\ldots$ & $\ldots$ \\
\hline XX Per & IRAS $01597+5459$ & 02:03:09.36 & $55: 13: 56.6$ & $\ldots$ & $n^{6}$ & $\ldots$ \\
\hline HD 236947 & IRAS $02036+5832$ & 02:07:12.06 & $58: 47: 15.9$ & $\ldots$ & $\ldots$ & $\ldots$ \\
\hline KK Per & IRAS $02068+5619$ & 02:10:15.79 & $56: 33: 32.7$ & $\ldots$ & $\ldots$ & $\ldots$ \\
\hline V550 Per & IRAS $02116+5754$ & $02: 15: 13.30$ & $58: 08: 32.3$ & $\ldots$ & $\ldots$ & $\ldots$ \\
\hline PP Per & IRAS $02135+5817$ & $02: 17: 08.23$ & $58: 31: 47.0$ & $\ldots$ & $\ldots$ & $\ldots$ \\
\hline BU Per & IRAS $02153+5711$ & 02:18:53.30 & $57: 25: 16.8$ & $\ldots$ & $n^{6}$ & $\ldots$ \\
\hline AD Per & IRAS $02169+5645$ & 02:20:29.00 & $56: 59: 35.2$ & $\ldots$ & $\ldots$ & $\ldots$ \\
\hline SU Per & IRAS $02185+5622$ & 02:22:06.89 & $56: 36: 14.9$ & $\ldots$ & $\ldots$ & $\ldots$ \\
\hline RS Per & IRAS $02188+5652$ & $02: 22: 24.30$ & $57: 06: 34.4$ & $\ldots$ & $\mathrm{n}^{6}$ & $\ldots$ \\
\hline V439 Per & IRAS $02196+5658$ & $02: 23: 11.03$ & $57: 11: 58.3$ & $\ldots$ & $\ldots$ & $\ldots$ \\
\hline V403 Per & $\ldots$ & $02: 23: 24.11$ & $57: 12: 43.1$ & $\ldots$ & $\ldots$ & $\ldots$ \\
\hline V441 Per & IRAS $02217+5712$ & $02: 25: 21.86$ & $57: 26: 14.1$ & $\ldots$ & $\ldots$ & $\ldots$ \\
\hline YZ Per & IRAS $02347+5649$ & $02: 38: 25.42$ & $57: 02: 46.2$ & $\ldots$ & $n^{6}$ & $\ldots$ \\
\hline GP Cas & IRAS $02360+5922$ & 02:39:50.44 & $59: 35: 51.3$ & $\ldots$ & $\ldots$ & $\ldots$ \\
\hline V648 Cas & IRAS $02473+5738$ & 02:51:03.95 & $57: 51: 19.9$ & $\ldots$ & $\ldots$ & $\ldots$ \\
\hline \multicolumn{7}{|c|}{ Mc8 } \\
\hline Mc8-01 & IRAS $18258-1058$ & $18: 28: 35.32$ & $-10: 56: 36.6$ & $y^{3}$ & $\mathrm{n}^{3}$ & $\ldots$ \\
\hline Mc8-02 & $\ldots$ & $18: 28: 39.13$ & $-10: 55: 52.7$ & $n^{3}$ & $n^{3}$ & $\ldots$ \\
\hline Mc8-03 & $\ldots$ & $18: 28: 52.08$ & $-10: 57: 57.7$ & $\mathrm{y}^{3}$ & $n^{3}$ & $\ldots$ \\
\hline Mc8-04 & $\ldots$ & $18: 28: 54.04$ & $-10: 56: 45.5$ & $n^{3}$ & $n^{3}$ & $\ldots$ \\
\hline Mc8-05 & $\ldots$ & $18: 28: 58.46$ & $-10: 56: 07.0$ & $y^{3}$ & $n^{3}$ & $\ldots$ \\
\hline Mc8-06 & $\ldots$ & 18:29:03.05 & $-10: 53: 15.4$ & $n^{3}$ & $n^{3}$ & $\ldots$ \\
\hline
\end{tabular}

References. ${ }^{1}$ Nakashima \& Deguchi (2006); ${ }^{2}$ Davies et al. (2008); ${ }^{3}$ Deguchi et al. (2010); ${ }^{4}$ Wilson \& Barrett (1972); ${ }^{5}$ Jiang et al. (1996); ${ }^{6}$ Takaba et al. (2001); ${ }^{7}$ Cho et al. (1996); ${ }^{8}$ Palagi et al. (1993); ${ }^{9}$ Szymczak et al. (2010); ${ }^{10}$ Jiang et al. (1999). 
Table 10

Summary of Previous Maser Observations of other Clusters 2

\begin{tabular}{|c|c|c|c|}
\hline Cluster & Line & Detection/No. of RSGs Observed & Telescope and Reference \\
\hline \multirow[t]{4}{*}{ RSGC1 } & $\mathrm{SiO} v=1, J=1-0$ & $4 / 13$ & $\mathrm{NRO} 45 \mathrm{~m}^{1}$ \\
\hline & $\mathrm{SiO} v=2, J=1-0$ & $1 / 13$ & $\mathrm{NRO} 45 \mathrm{~m}^{1}$ \\
\hline & $\mathrm{H}_{2} \mathrm{O}\left(6_{16}-5_{23}\right)$ & $1 / 14$ & $\mathrm{NRO} 45 \mathrm{~m}^{1}$ \\
\hline & $\mathrm{OH}^{2} \Pi_{3 / 2} J=3 / 2 F=1-2$ & $1 / 14$ & $\mathrm{VLA}^{2}$ \\
\hline \multirow[t]{4}{*}{ RSGC2 } & $\mathrm{SiO} v=1, J=1-0$ & $1 / 5$ & $\mathrm{NRO} 45 \mathrm{~m}^{3}$ \\
\hline & $\mathrm{SiO} v=2, J=1-0$ & $0 / 5$ & $\mathrm{NRO} 45 \mathrm{~m}^{3}$ \\
\hline & $\mathrm{H}_{2} \mathrm{O}\left(6_{16}-5_{23}\right)$ & $1 / 5$ & $\mathrm{NRO} 45 \mathrm{~m}^{3}$ \\
\hline & $\mathrm{OH}^{2} \Pi_{3 / 2} J=3 / 2 F=1-2$ & $0 / 1$ & NRAO $43 m^{4}$ \\
\hline \multirow[t]{3}{*}{ RSGC2 SW } & $\mathrm{SiO} v=1, J=1-0$ & $3 / 5$ & $\mathrm{NRO} 45 \mathrm{~m}^{3}$ \\
\hline & $\mathrm{SiO} v=2, J=1-0$ & $2 / 5$ & $\mathrm{NRO} 45 \mathrm{~m}^{3}$ \\
\hline & $\mathrm{H}_{2} \mathrm{O}\left(6_{16}-5_{23}\right)$ & $3 / 5$ & $\mathrm{NRO} 45 \mathrm{~m}^{3}$ \\
\hline \multirow[t]{6}{*}{ Per OB1 } & $\mathrm{SiO} v=1, J=1-0$ & $2 / 7$ & $\mathrm{NRO} 45 \mathrm{~m}^{5,6,7}$ \\
\hline & $\mathrm{SiO} v=2, J=1-0$ & $1 / 7$ & $\mathrm{NRO} 45 \mathrm{~m}^{5,6,7}$ \\
\hline & $\mathrm{SiO} v=3, J=1-0$ & $0 / 1$ & $\mathrm{NRO} 45 \mathrm{~m}^{6}$ \\
\hline & $\mathrm{H}_{2} \mathrm{O}\left(6_{16}-5_{23}\right)$ & $1 / 7$ & Medicina $32 \mathrm{~m}^{8}, \mathrm{KSRC} 4 \mathrm{~m}^{9}$ \\
\hline & $\mathrm{OH}^{2} \Pi_{3 / 2} J=3 / 2 F=1-2$ & $1 / 1$ & $\mathrm{NRT}^{10}$ \\
\hline & $\mathrm{OH}^{2} \Pi_{3 / 2} J=3 / 2 F=2-2$ & $1 / 1$ & $\mathrm{NRT}^{10}$ \\
\hline \multirow[t]{3}{*}{$\mathrm{Mc} 8$} & $\mathrm{SiO} v=1, J=1-0$ & $3 / 6$ & $\mathrm{NRO} 45 \mathrm{~m}^{3}$ \\
\hline & $\mathrm{SiO} v=2, J=1-0$ & $3 / 6$ & $\mathrm{NRO} 45 \mathrm{~m}^{3}$ \\
\hline & $\mathrm{H}_{2} \mathrm{O}\left(6_{16}-5_{23}\right)$ & $0 / 6$ & $\mathrm{NRO} 45 \mathrm{~m}^{3}$ \\
\hline
\end{tabular}

Notes. NRO45m: 45 m radio telescope, Nobeyama Radio Observatory; VLA: The Very Large Array, National Radio Astronomy Observatory; Medicina32m: Medicina 32 m Antenna, Istituto di Radioastronomia; KSRC34m: Kashima 34 m Antenna, Kashima Space Research Center; NRT: Nançay radio telescope, Observatoire de Paris; NRAO43m: $43 \mathrm{~m}$ radio telescope, National Radio Astronomy Observatory.

References. ${ }^{1}$ Nakashima \& Deguchi (2006); ${ }^{2}$ Davies et al. (2008); ${ }^{3}$ Deguchi et al. (2010); ${ }^{4}$ Wilson \& Barrett (1972); ${ }^{5}$ Jiang et al. (1996); ${ }^{6}$ Cho et al. (1996); ${ }^{7}$ Jiang et al. (1999); ${ }^{8}$ Palagi et al. (1993); ${ }^{9}$ Takaba et al. (2001);

10 Szymczak et al. (2010).

in Appendix B. Similar to the analysis on $\mathrm{Wd1}$, we adopted extinction corrections based on the extinction coefficients given in literature (Indebetouw et al. 2005; Levesque et al. 2005; Davies et al. 2007, 2008). As we mentioned in Section 5.1, some authors recently use a slightly different formula for the conversion of the extinction values: we used $A_{K}=1.8 \times E_{H-K}$, while some authors recently use $A_{K}=1.5 \times E_{H-K}$ (see, e.g., Nishiyama et al. 2006). We numerically evaluated the difference between the results obtained by the two formulae: for the majority of the samples with $A_{K}<2.5$ (this $A_{K}$ is based on the values given in Table 11), the relative difference in the finally derived luminosities is less than $20 \%$. For exceptional sources with a large extinction $\left(A_{K}>3.0\right)$, the relative difference occasionally goes beyond $40 \%$, but such objects are quite rare (only two). Therefore, the selection of the conversion formula does not affect the results of our discussion. In Figure 4, we present the distribution of 29 RSGs in six clusters (including Wd1 $)$ on the two-color diagram of $\log \left(F_{21} / F_{12}\right)$ and [K-12.13] colors. Although five RSGs in Per OB1 (the red open diamonds) exhibit relatively small [K-12.13] values, there seems to be no other significant inhomogeneity in the distribution (since RSGs in Per OB1 have been identified mainly in optical (Humphreys 1970), the sample could be somewhat biased). Then, absolute luminosities and mass-loss rates were estimated using the same methodology as we adopted for Wd1. The obtained absolute luminosity and the best-fit parameters of DUSTY models are summarized in Table 8. Then, the results of previous maser observations of 53 RSGs in five clusters are summarized in Tables 9 and 10.

Figure 5 shows the two-color diagrams of RSGs in five clusters and Wd1. The data points in Figure 5 are classified into either detections (red filled circles) or non-detections (blue

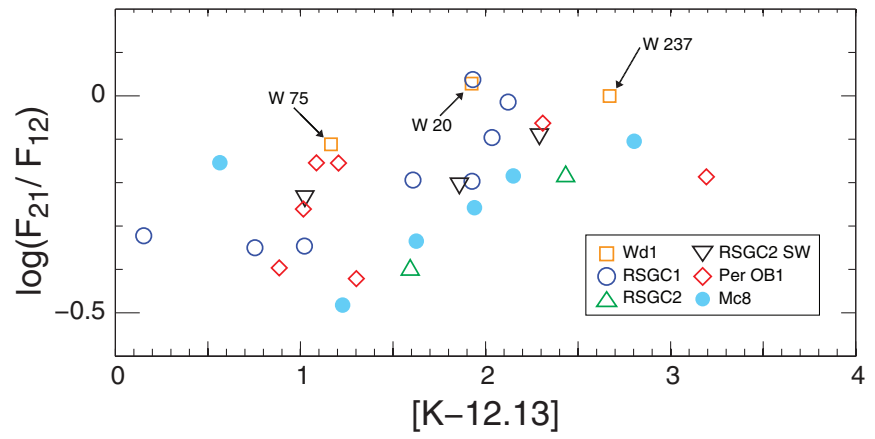

Figure 4. Distribution of RSGs in six massive clusters on the infrared two-color diagram. The member RSGs of Wd1 are labeled with the arrows (W 26 is not indicated due to a large uncertainty in its $K$-band flux $)$. In the $\log \left(F_{21} / F_{12}\right)$ color, $F_{21}$ and $F_{12}$ represent the MSX $21.34 \mu \mathrm{m}$ and $12.13 \mu \mathrm{m}$ fluxes, respectively. The $[K-12.13]$ color is defined by $[K-12.13]=K+2.5 \log \left(F_{12} / 26.51[\mathrm{Jy}]\right)$; here, $K$ and $F_{12}$ are the 2MASS $K$-band magnitude and the $M S X 12.13 \mu \mathrm{m}$ flux. The interstellar extinction corrections were adopted (see Sections 5.1 and 5.2). (A color version of this figure is available in the online journal.)

open circles) in each maser line. In these two-color diagrams, we clearly see that the distributions of maser detections and nondetections are different in colors, i.e., detections are distributed in the upper right of each panel, while the non-detections are distributed in lower left of each panel. Since generally $\log \left(F_{21} / F_{12}\right)$ and $[\mathrm{K}-12.13]$ are related to dust temperature and mid-infrared optical depth of the envelopes, respectively (see, e.g., Le Sidaner \& Le Bertre 1996), the concentration of maser detections suggests that maser emission is selectively detected toward RSGs with a low dust temperature and large optical depth. On the other hand, we cannot see a clear difference 


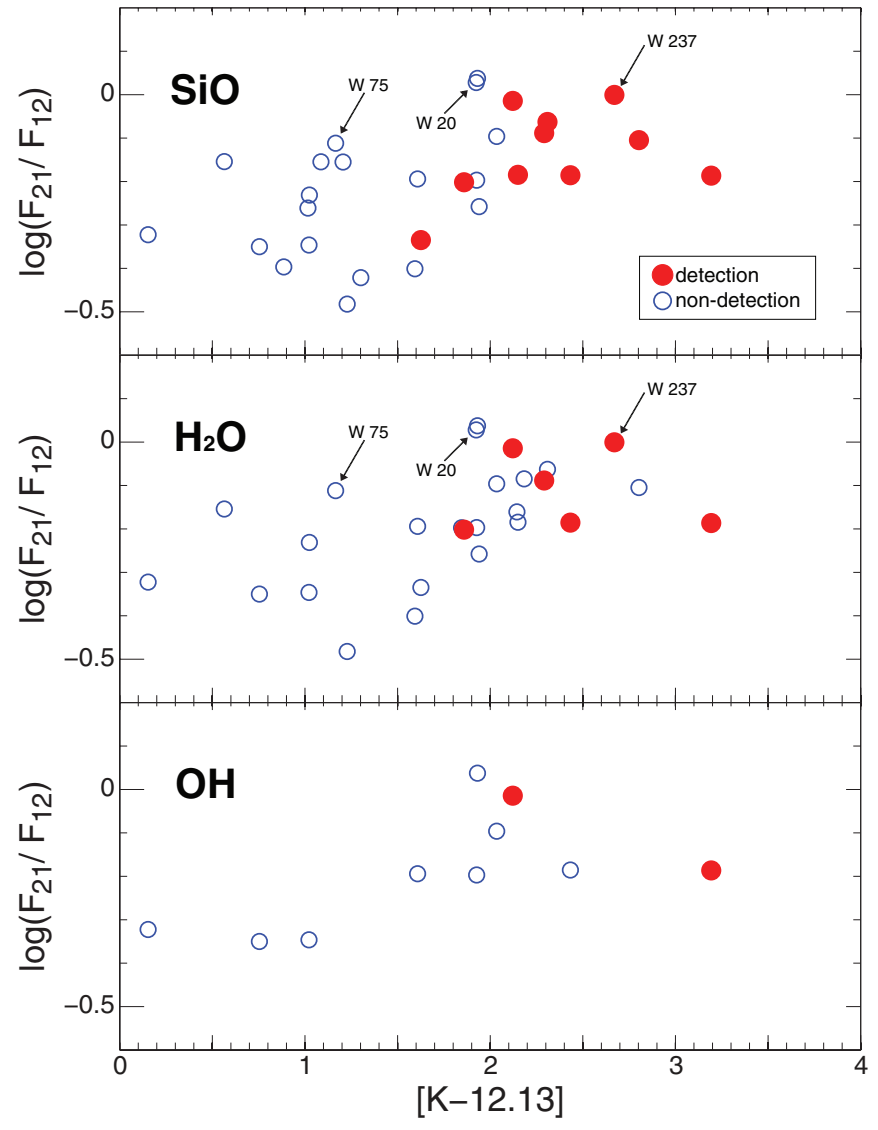

Figure 5. Distributions of detections and non-detections of maser observations in the $\mathrm{SiO}(J=1-0, v=1$ and 2$), \mathrm{H}_{2} \mathrm{O}(22 \mathrm{GHz})$, and $\mathrm{OH}$ maser $(1612 \mathrm{MHz})$ lines on the infrared two-color diagrams. The definitions of colors are the same as Figure 4. The red filled and blue open circles represent detections and nondetections. The member RSGs of Wd1 are indicated by the arrows. W 26 is not indicated in this plot due to large uncertainty in its photometric value.

(A color version of this figure is available in the online journal.)

between the $\mathrm{SiO}, \mathrm{H}_{2} \mathrm{O}$, and $\mathrm{OH}$ maser lines in the distributions of detected and non-detected sources (even though the number of $\mathrm{OH}$ maser observations is not sufficient enough for comparison).

Interestingly, however, one may point out a weak but possible difference between the $\mathrm{SiO}, \mathrm{H}_{2} \mathrm{O}$, and $\mathrm{OH}$ maser lines in the distributions of detections and non-detections in the $\log \dot{M}$ versus $\log L$ plot given in Figure 6 (Note that in this figure we do not intend to discuss the correlation between the massloss rate and absolute luminosity; it is natural that there is a correlation between $\dot{M}$ and $L$ in Figure 6, because both values are derived from the same SED. The main focus here is only the distributions of detections and non-detections in mass-loss rates and absolute luminosities). The detections in the $\mathrm{SiO}$ line distribute in a wide range of $L$, whereas the majority of the $\mathrm{H}_{2} \mathrm{O}$ maser detections tend to locate in a region with large $L$ (above, roughly $10^{5} L_{\odot}$ ) except for one data point (St2-08) around $\log L \sim 4.55$. Of course, we need to bear in mind that the distance includes a relatively large uncertainty, and the number of the samples is not large enough. However, as a possibility, one may interpret this difference for astrophysical reasons; for example, difference in excitation conditions of masers, such as, difference in excitation temperatures and critical densities. And if this difference is originated in the intrinsic astrophysical reasons, one may expect that maser lines are used to select a particular group of RSGs lying in a small range of mass-loss rate and luminosity. On the $\mathrm{OH}$ maser observation, at the moment we

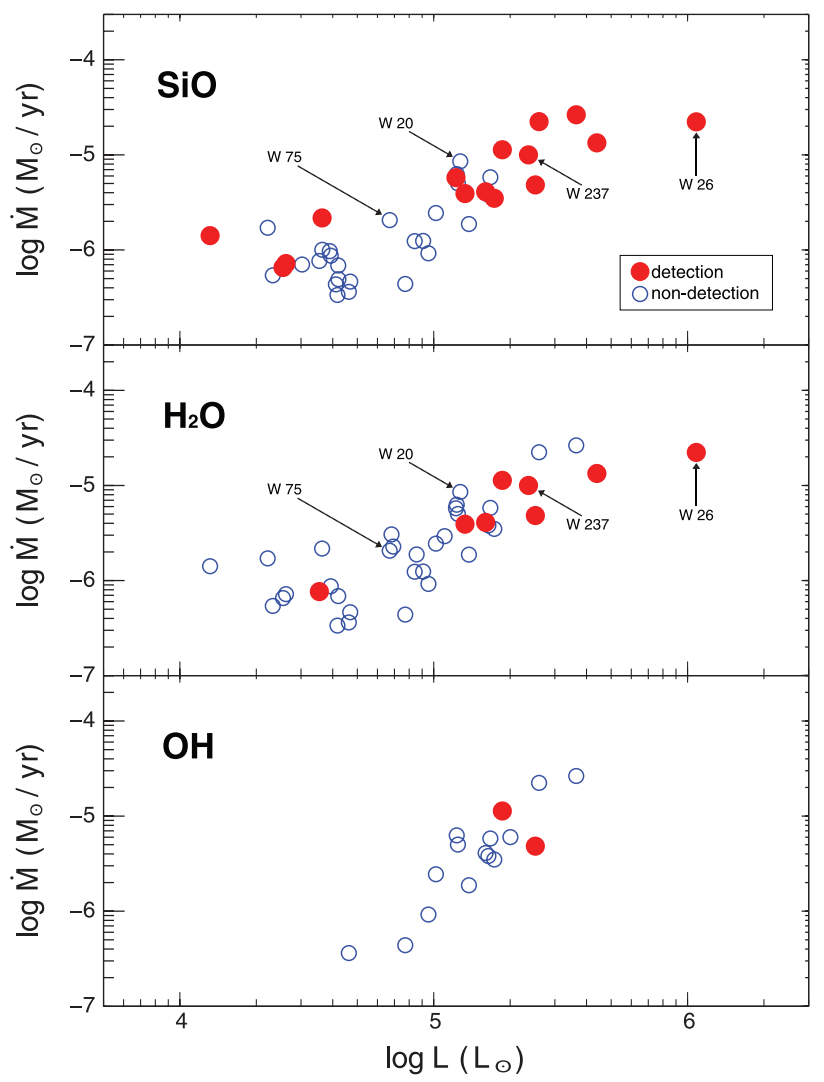

Figure 6. Distributions of detections and non-detections of maser observations in the $\mathrm{SiO}(J=1-0, v=1$ and 2$), \mathrm{H}_{2} \mathrm{O}(22 \mathrm{GHz})$, and $\mathrm{OH}$ maser $(1612 \mathrm{MHz})$ lines on the mass-loss rate vs. absolute luminosity diagram. The red filled and blue open circles represent detections and non-detections. The member RSGs of $\mathrm{Wd} 1$ are indicated by the arrows.

(A color version of this figure is available in the online journal.)

cannot see any tendency, because the number of observations is clearly too small to comment on. Since the physical conditions required for pumping $\mathrm{OH}$ maser are also different from those for $\mathrm{SiO}$ and $\mathrm{H}_{2} \mathrm{O}$ masers, one may expect that there will be a recognizable difference from $\mathrm{SiO}$ and $\mathrm{H}_{2} \mathrm{O}$ masers if the number of $\mathrm{OH}$ observations is increased.

Using the photometric data collected, we inspected the distribution of detections and non-detections of maser searches on the $Q 1-Q 2$ diagram, which has been suggested by Messineo et al. (2012). Messineo et al. (2012) proposed a new source selection method for identifying mass-losing evolved stars, which is based on photometric data from 2MASS $(J, H$, $K$-band magnitudes) and GLIMPSE ( $8.0 \mu \mathrm{m}$ band magnitude) and on the $Q 1$ and $Q 2$ parameters. These two parameters are independent of interstellar extinction. The $Q 1$ parameter [ $Q 1=$ $(J-H)-1.8 \times(H-K)]$ is a measure of the deviation from the reddening vector in the $J-H$ versus $H-K$ plane. Early-type stars have $Q 1$ values around $1, \mathrm{~K}$-giants around $0.4 \mathrm{mag}$, and dusty circumstellar envelopes of evolved stars generate even smaller $Q 1$ values. On average, higher mass-loss rates produce smaller $Q 1$ values (Messineo et al. 2012). Similarly, the $Q 2$ parameter $(Q 2=(J-K)-2.69 \times(K-[8.0])$; here, [8.0] means the GLIMPSE $8 \mu \mathrm{m}$ band magnitude) measures the deviation from the reddening vector in the plane $J-K$ versus $K-$ [8.0]. On average, higher mass-loss rates imply smaller $Q 2$ values as well as $Q 1$ (Messineo et al. 2012).

In Figure 7, we present the distribution of the present RSG samples on the $Q 1-Q 2$ diagram (red and blue marks); 


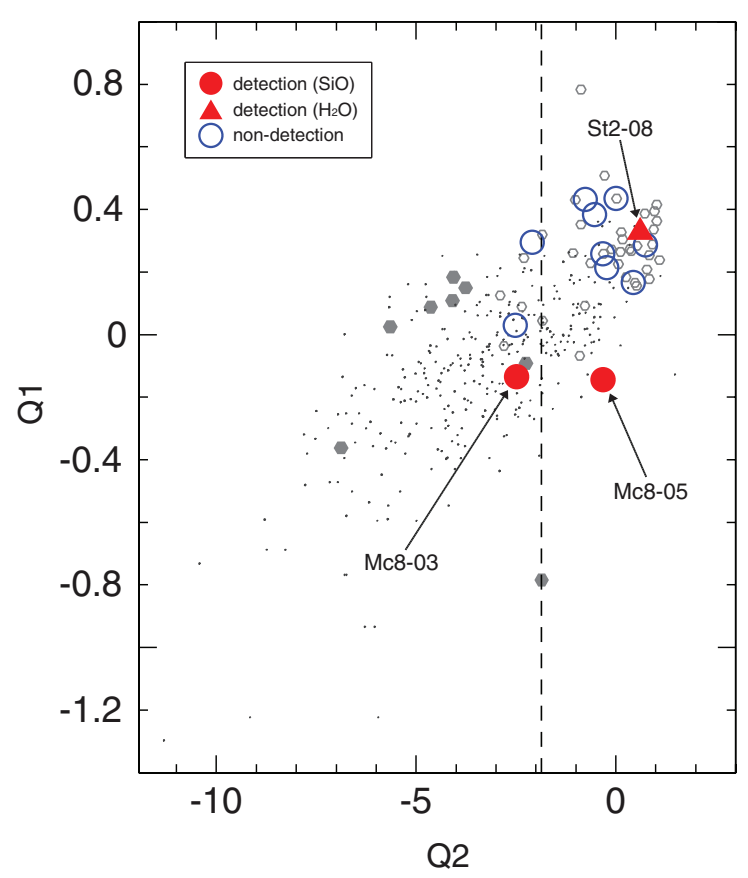

Figure 7. Distributions of detections and non-detections of maser observations on the extinction-free $Q 1$ and $Q 2$ parameters overlaid on a selected region of Figure 3 in Verheyen et al. (2012). The red filled circles represent RSGs detected only in the $\mathrm{SiO}$ maser line (and both $\mathrm{H}_{2} \mathrm{O}$ and $\mathrm{OH}$ maser searches are negative). The red filled triangle represents RSG detected only in the $\mathrm{H}_{2} \mathrm{O}$ maser line (and both $\mathrm{SiO}$ and $\mathrm{OH}$ maser searches are negative). The open blue circles represent the RSGs that are negative in all three maser lines $\left(\mathrm{SiO}, \mathrm{H}_{2} \mathrm{O}\right.$, and $\left.\mathrm{OH}\right)$. The gray filled and empty hexagons represent RSGs with and without maser detections, respectively (Verheyen et al. 2012). The small dots represent $\mathrm{SiO}$ maser detections of Mira-like AGB stars (Messineo et al. 2002). The vertical dotted line represents the Q2 value of -1.9 (see the text). RSGs detected in maser lines are indicated by arrows along with object names.

(A color version of this figure is available in the online journal.)

the samples of Mira-like AGB stars (Messineo et al. 2002) and Galactic RSGs (Verheyen et al. 2012) are also plotted together (gray marks) for comparison. Unfortunately, only 13 data points of the present samples can be plotted on the diagram, because for the majority of stars the GLIMPSE $8 \mu \mathrm{m}$ data are not available due to source confusion (the present RSG samples are taken from star clusters, in which RSGs roll up in a small region). Nevertheless, as suggested by Verheyen et al. (2012), we see a clear tendency in the diagram: the detections in the $\mathrm{SiO} J=1-0$ lines exhibit negative $Q 1$ values, while non-detections exhibit relatively larger $Q 1$ values. Although we see the detection in the $\mathrm{H}_{2} \mathrm{O}$ maser line $(\mathrm{St} 2-08)$ at $(Q 1, Q 2)=(0.33,0.61)$, this presumably suggests that the $\mathrm{SiO}$ maser line is more sensitive to the $Q$-parameters than the $\mathrm{H}_{2} \mathrm{O}$ maser line ( $\mathrm{St} 2-08$ is negative in the $\mathrm{SiO}$ maser search; Deguchi et al. 2010). In the previous work by Verheyen et al. (2012) on Galactic RSG samples, the detections in the $\mathrm{SiO} J=2-1$ line are lying below $Q 2=-1.9$. However, in the present case we find one detection (Mc8-05) above $Q 2=-1.9$. In any case, a larger statistic is required to confirm the suggested boundary on the $Q 1-Q 2$ diagram.

\section{SUMMARY}

In this paper, we have reported the result of an ATCA observation of the Wd1 region in the $\mathrm{SiO} v=1, J=1-0$, and $\mathrm{H}_{2} \mathrm{O} 6_{16}-5_{23}$ lines. We analyzed infrared SEDs of 57 RSGs in six clusters including $\mathrm{Wd} 1$ to investigate the correlations between maser and infrared properties. The main results of this research are summarized below.
1. The $\mathrm{SiO} v=1, J=1-0$, and $\mathrm{H}_{2} \mathrm{O} 6_{16}-5_{23}$ lines are detected toward two of four known RSGs in Wd1, and the large velocity ranges of detected maser lines are consistent with the RSG status. The velocity ranges of the $\mathrm{SiO}$ maser line are wider than that of the $\mathrm{H}_{2} \mathrm{O}$ line; this fact may suggest that the RSG envelopes are deviated from a spherical symmetry.

2. RSGs with maser emission seem to exhibit relatively large $\log \left(F_{21} / F_{12}\right)$ and [K-12.13] colors compared to nondetections. The mass-loss rates derived from dust radiative transfer modeling suggest that RSGs with maser emission exhibit a relatively large mass-loss rate compared to RSGs with no maser emission.

3. RSGs with $\mathrm{SiO}$ maser emission homogeneously distribute in $L$, whereas those with $\mathrm{H}_{2} \mathrm{O}$ maser emission tend to distribute in a region with large $L$ values (above, roughly $\left.4 \times 10^{4} L_{\odot}\right)$.

4. The distribution of the detections and non-detection on the $Q 1-Q 2$ diagram is roughly consistent with the previous study by Verheyen et al. (2012), even though one data point of an $\mathrm{SiO}$ maser detection is found above $Q 2=-1.9$.

This work is supported by grants awarded to Jun-ichi Nakashima from the Research Grants Council of Hong Kong (project code: HKU 703308P), the Seed Funding Program for Basic Research of the University of Hong Kong (project code: 200802159006), and the Small Project Funding of the University of Hong Kong (project code: 201007176004). The Australia Telescope Compact Array is part of the Australia Telescope National Facility which is funded by the Commonwealth of Australia for operation as a National Facility managed by CSIRO. The authors thank Ryszard Szczerba, Mikako Matsuura, and Hiroshi Imai for stimulating discussions and valuable comments.

\section{APPENDIX A}

\section{NEW SIO MASER DETECTION TOWARD IRAS 16105-4205}

We detected the $\mathrm{SiO} v=1, J=1-0$ line toward IRAS $16105-4205$ for the first time. The peak velocity and peak flux are $-83 \mathrm{~km} \mathrm{~s}^{-1}$ and $8.8 \mathrm{Jy}$, respectively. The velocity-integrated flux of the detection is $28.2 \mathrm{Jy} \mathrm{km} \mathrm{s}^{-1}$. The $\mathrm{SiO} v=1, J=2-1$ line has been detected previously by Haikala et al. (1994). In Figure 8 , we present the line profile of the $v=1, J=1-0$ line superimposed on that of the $v=1, J=2-1$ line (Haikala et al. 1994). IRAS 16105-4205 was previously classified as a

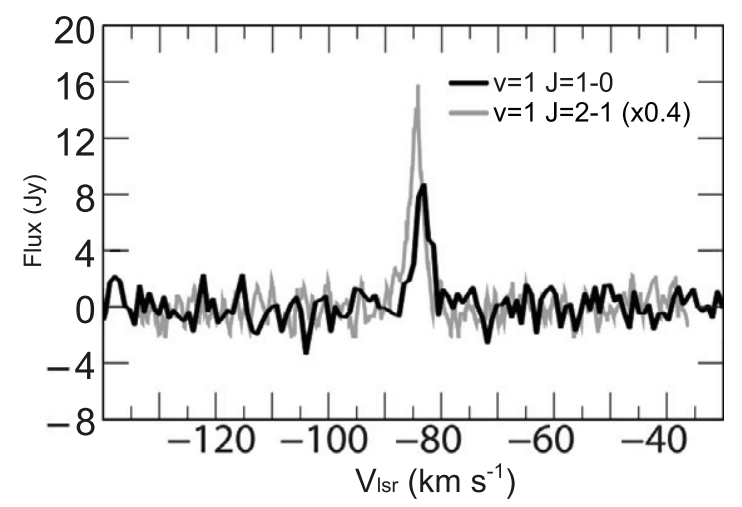

Figure 8. Spectrum of the $\mathrm{SiO} v=1, J=1-0$ line of IRAS 16105-4205 (thick line), superimposed on the spectrum of the $\mathrm{SiO} v=1, J=2-1$ line (gray line; Haikala et al. 1994). The $v=1, J=1-0$ line is detected for the first time. 
carbon star based on near-infrared photometric colors (Fouque et al. 1992). However, since the object is detected in $\mathrm{SiO}$ maser lines, we suspect that it may be an oxygen-rich star rather than a carbon star. In fact, Dollery et al. (1987) and Chen \& Gao (2002) respectively detected the $\mathrm{OH} 1612 \mathrm{MHz}$ maser emission and $9.7 \mu \mathrm{m}$ silicate feature toward this object, although an $\mathrm{H}_{2} \mathrm{O}$ maser search was negative (Deguchi et al. 1989).

\section{APPENDIX B}

\section{PHOTOMETRIC DATA OF RSGs ASSOCIATED WITH MASSIVE CLUSTERS}

Photometric data and extinction coefficients used for the analysis are summarized in Tables 11-15. SED diagrams and the results of DUSTY modeling are given in Figure 9.

Table 11

Infrared Photometric Data 1

\begin{tabular}{|c|c|c|c|c|c|c|c|c|c|}
\hline \multirow{2}{*}{$\begin{array}{l}\text { Source } \\
\text { Name }\end{array}$} & \multirow{2}{*}{$\begin{array}{c}A_{K} \\
2.17 \mu \mathrm{m} \\
(\mathrm{mag})\end{array}$} & \multicolumn{2}{|c|}{ DENIS $^{\mathrm{a}}$} & \multicolumn{6}{|c|}{$2 \mathrm{MASS}^{\mathrm{b}}$} \\
\hline & & $\begin{array}{c}I \\
(\mathrm{mag})\end{array}$ & $\begin{array}{c}\sigma_{I} \\
(\mathrm{mag})\end{array}$ & $\begin{array}{c}J \\
(\mathrm{mag})\end{array}$ & $\begin{array}{c}\sigma_{J} \\
(\mathrm{mag})\end{array}$ & $\begin{array}{c}H \\
(\mathrm{mag})\end{array}$ & $\begin{array}{c}\sigma_{H} \\
(\mathrm{mag})\end{array}$ & $\begin{array}{c}K \\
(\mathrm{mag})\end{array}$ & $\begin{array}{c}\sigma_{K} \\
(\mathrm{mag})\end{array}$ \\
\hline \multicolumn{10}{|c|}{ Wd1 } \\
\hline W 237 & 0.96 & 10.25 & 0.03 & 5.08: & 0.02 & 3.01 & 0.27 & 2.18 & 0.28 \\
\hline W 26 & 1.33 & 9.74 & 0.04 & 4.31 & 0.52 & 1.35 & 1.32 & $6.21 \dagger$ & $\ldots$ \\
\hline W 20 & 1.55 & 13.05 & 0.03 & 6.38 & 0.02 & 4.38: & 0.08 & 2.61 & 0.41 \\
\hline W 75 & 1.47 & 14.03 & 0.03 & 6.93 & 0.02 & 4.67: & 0.02 & 3.28 & 0.27 \\
\hline \multicolumn{10}{|c|}{ RSGC1 } \\
\hline F01 & 2.58 & $\ldots$ & $\ldots$ & 9.75 & 0.03 & 6.59 & 0.02 & 4.96 & 0.02 \\
\hline F02 & 2.83 & $\ldots$ & $\ldots$ & 9.90 & 0.03 & 6.70 & 0.02 & 5.03 & 0.02 \\
\hline F03 & 2.46 & $\ldots$ & $\ldots$ & 9.95 & 0.03 & 6.92 & 0.02 & 5.33 & 0.02 \\
\hline F04 & 2.46 & $\ldots$ & $\ldots$ & 9.66 & 0.03 & 6.80 & 0.02 & 5.34 & 0.02 \\
\hline F05 & 2.77 & $\ldots$ & $\ldots$ & 10.55 & 0.03 & 7.18 & 0.03 & 5.54 & 0.03 \\
\hline F06 & 2.19 & $\ldots$ & $\ldots$ & 9.87 & 0.03 & 7.04 & 0.03 & 5.61 & 0.03 \\
\hline F07 & 2.33 & $\ldots$ & $\ldots$ & 9.94 & 0.03 & 7.07 & 0.03 & 5.63 & 0.02 \\
\hline F08 & 2.84 & $\ldots$ & $\ldots$ & 10.77 & 0.03 & 7.33 & 0.02 & 5.65 & 0.02 \\
\hline F09 & 2.44 & $\ldots$ & $\ldots$ & 10.26 & 0.03 & 7.24 & 0.02 & 5.67 & 0.02 \\
\hline F10 & 2.45 & 18.47 & 0.29 & 10.18 & 0.03 & 7.22 & 0.03 & 5.71 & 0.02 \\
\hline F11 & 2.63 & $\ldots$ & $\ldots$ & 10.47 & 0.03 & 7.33 & 0.03 & 5.72 & 0.02 \\
\hline F12 & 2.57 & $\ldots$ & $\ldots$ & 10.14 & 0.03 & 7.24 & 0.02 & 5.86 & 0.03 \\
\hline F13 & 3.19 & $\ldots$ & $\ldots$ & 10.91 & 0.03 & 7.72 & 0.02 & 5.96 & 0.02 \\
\hline F14 & 2.29 & $\ldots$ & $\ldots$ & 10.50 & 0.03 & 7.58 & 0.02 & 6.17 & 0.02 \\
\hline \multicolumn{10}{|c|}{ RSGC2 } \\
\hline St2-01 & 1.45 & 13.92 & 0.03 & 7.82 & 0.02 & 6.02 & 0.03 & 5.11 & 0.02 \\
\hline St2-02 & 1.42 & 14.35 & 0.03 & 8.35 & 0.03 & 6.21 & 0.03 & 5.26 & 0.02 \\
\hline St2-03 & 1.39 & 12.66 & 0.03 & 6.90 & 0.02 & 5.05 & 0.02 & 4.12 & 0.27 \\
\hline St2-04 & 1.34 & 12.85 & 0.03 & 7.27 & 0.02 & 5.46 & 0.03 & 4.50: & 0.04 \\
\hline St2-05 & 1.42 & 13.94 & 0.03 & 8.22 & 0.02 & 6.21 & 0.02 & 5.24 & 0.02 \\
\hline \multicolumn{10}{|c|}{ RSGC2 SW } \\
\hline St2-08 & 1.64 & 15.19 & 0.05 & 8.57 & 0.02 & 6.31 & 0.02 & 5.23 & 0.02 \\
\hline St2-11 & 4.58 & $\ldots$ & $\ldots$ & 14.23 & 0.04 & 9.92 & 0.02 & 7.32 & 0.02 \\
\hline St2-14 & 1.99 & 16.03 & 0.07 & 8.53 & 0.02 & 6.05 & 0.03 & 4.82 & 0.02 \\
\hline St2-18 & 1.87 & 14.56 & 0.04 & 7.15 & 0.03 & 4.70 & 0.02 & 2.90 & 0.19 \\
\hline St2-26 & 2.52 & $\ldots$ & $\ldots$ & 12.03 & 0.04 & 8.52 & 0.03 & $6.95 \dagger$ & $\ldots$ \\
\hline \multicolumn{10}{|c|}{ Per OB1 } \\
\hline W Per & 0.45 & $\ldots$ & $\ldots$ & 3.10 & 0.20 & 2.00 & 0.17 & 1.57 & 0.25 \\
\hline S Per & 0.47 & $\ldots$ & $\ldots$ & 2.95 & 0.28 & 1.85 & 0.24 & 1.12 & 0.22 \\
\hline T Per & 0.35 & $\ldots$ & $\ldots$ & 4.03 & 0.25 & 3.02 & 0.17 & 2.58 & 0.24 \\
\hline V605 Cas & 0.35 & $\ldots$ & $\ldots$ & 3.95 & 0.26 & 3.04 & 0.20 & 2.48 & 0.27 \\
\hline V778 Cas & 0.19 & $\ldots$ & $\ldots$ & 4.03 & 0.30 & 2.97 & 0.21 & 2.49 & 0.32 \\
\hline PR Per & 0.28 & $\ldots$ & $\ldots$ & 3.56 & 0.31 & 2.68 & 0.19 & 2.25 & 0.30 \\
\hline FZ Per & 0.22 & $\ldots$ & $\ldots$ & 3.80 & 0.25 & 2.91 & 0.20 & 2.48 & 0.24 \\
\hline BD+59 372 & 0.28 & $\ldots$ & $\ldots$ & 5.33 & 0.25 & 4.20 & 0.23 & 3.74 & 0.26 \\
\hline XX Per & 0.31 & $\ldots$ & $\ldots$ & 3.44 & 0.26 & 2.48 & 0.23 & 1.97 & 0.24 \\
\hline HD 236947 & 0.31 & $\ldots$ & $\ldots$ & 4.02 & 0.23 & 3.13 & 0.19 & 2.62 & 0.24 \\
\hline KK Per & 0.31 & $\ldots$ & $\ldots$ & 3.00 & 0.20 & 2.14 & 0.17 & 1.68 & 0.21 \\
\hline V550 Per & 0.31 & $\ldots$ & $\ldots$ & 4.72 & 0.27 & 3.66 & 0.20 & 3.19 & 0.32 \\
\hline PP Per & 0.31 & $\ldots$ & $\ldots$ & 4.57 & 0.25 & 3.52 & 0.22 & 2.95 & 0.24 \\
\hline BU Per & 0.36 & $\ldots$ & $\ldots$ & 3.68 & 0.29 & 2.68 & 0.20 & 2.19 & 0.23 \\
\hline AD Per & 0.31 & $\ldots$ & $\ldots$ & 3.38 & 0.24 & 2.48 & 0.21 & 1.94 & 0.26 \\
\hline SU Per & 0.23 & $\ldots$ & $\ldots$ & 2.82 & 0.25 & 1.93 & 0.18 & 1.46 & 0.22 \\
\hline RS Per & 0.29 & $\ldots$ & $\ldots$ & 3.05 & 0.22 & 2.11 & 0.19 & 1.56 & 0.21 \\
\hline
\end{tabular}


Table 11

(Continued)

\begin{tabular}{|c|c|c|c|c|c|c|c|c|c|}
\hline \multirow{2}{*}{$\begin{array}{l}\text { Source } \\
\text { Name }\end{array}$} & \multirow{2}{*}{$\begin{array}{c}A_{K} \\
2.17 \mu \mathrm{m} \\
(\mathrm{mag})\end{array}$} & \multicolumn{2}{|c|}{ DENIS $^{\mathrm{a}}$} & \multicolumn{6}{|c|}{$2 \mathrm{MASS}^{\mathrm{b}}$} \\
\hline & & $\begin{array}{c}I \\
(\mathrm{mag})\end{array}$ & $\begin{array}{c}\sigma_{I} \\
(\mathrm{mag})\end{array}$ & $\begin{array}{c}J \\
(\mathrm{mag})\end{array}$ & $\begin{array}{c}\sigma_{J} \\
(\mathrm{mag})\end{array}$ & $\begin{array}{c}H \\
(\mathrm{mag})\end{array}$ & $\begin{array}{c}\sigma_{H} \\
(\mathrm{mag})\end{array}$ & $\begin{array}{c}K \\
(\mathrm{mag})\end{array}$ & $\begin{array}{c}\sigma_{K} \\
(\mathrm{mag})\end{array}$ \\
\hline V439 Per & 0.21 & $\ldots$ & $\ldots$ & 4.13 & 0.29 & 3.22 & 0.23 & 2.69 & 0.30 \\
\hline V403 Per & 0.24 & $\ldots$ & $\ldots$ & 4.48 & 0.27 & 3.46 & 0.22 & 2.99 & 0.29 \\
\hline V441 Per & 0.28 & $\ldots$ & $\ldots$ & 3.47 & 0.26 & 2.47 & 0.22 & 2.04 & 0.24 \\
\hline YZ Per & 0.26 & $\ldots$ & $\ldots$ & 3.26 & 0.26 & 2.30 & 0.23 & 1.91 & 0.26 \\
\hline GP Cas & 0.31 & $\ldots$ & $\ldots$ & 3.69 & 0.27 & 2.44 & 0.21 & 1.94 & 0.24 \\
\hline V648 Cas & 0.45 & $\ldots$ & $\ldots$ & 3.83 & 0.34 & 2.71 & 0.22 & 2.11 & 0.27 \\
\hline \multicolumn{10}{|c|}{ Mc8 } \\
\hline Mc8-01 & 1.05 & 16.37 & 0.09 & 7.90 & 0.03 & 5.98 & 0.03 & 4.88 & 0.02 \\
\hline Mc8-02 & 0.88 & 13.41 & 0.05 & 7.74 & 0.02 & 5.86 & 0.04 & 4.97 & 0.02 \\
\hline Mc8-03 & 1.40 & $\ldots$ & $\ldots$ & 9.78 & 0.02 & 7.54 & 0.04 & 6.23 & 0.02 \\
\hline Mc8-04 & 1.51 & $\ldots$ & $\ldots$ & 9.30 & 0.02 & 6.90 & 0.04 & 5.59 & 0.02 \\
\hline Mc8-05 & 1.24 & 17.44 & 0.14 & 8.88 & 0.04 & 6.80 & 0.04 & 5.57 & 0.02 \\
\hline Mc8-06 & 0.59 & 11.06 & 0.02 & 6.84 & 0.03 & 5.26 & 0.03 & 4.49 & 0.02 \\
\hline
\end{tabular}

Notes.

${ }^{a}$ For saturating sources, the measured fluxes are calibrated by PSF fitting.

b For saturating sources, the measured fluxes are calibrated by one-dimensional radial profiles.

: The colon represents uncertain detection.

$\dagger$ The dagger represents $95 \%$ confidence upper limit.

Table 12

Infrared Photometric Data 2

\begin{tabular}{|c|c|c|c|c|c|c|c|c|}
\hline \multirow{2}{*}{$\begin{array}{l}\text { Source } \\
\text { Name }\end{array}$} & \multicolumn{8}{|c|}{$W I S E^{\mathrm{a}}$} \\
\hline & $\begin{array}{c}3.4 \mu \mathrm{m} \\
(\mathrm{mag})\end{array}$ & $\begin{array}{c}\sigma_{3.4 \mu \mathrm{m}} \\
(\mathrm{mag})\end{array}$ & $\begin{array}{c}4.6 \mu \mathrm{m} \\
(\mathrm{mag})\end{array}$ & $\begin{array}{c}\sigma_{4.6 \mu \mathrm{m}} \\
(\mathrm{mag})\end{array}$ & $\begin{array}{l}12 \mu \mathrm{m} \\
(\mathrm{mag})\end{array}$ & $\begin{array}{c}\sigma_{12 \mu \mathrm{m}} \\
(\mathrm{mag})\end{array}$ & $\begin{array}{l}22 \mu \mathrm{m} \\
(\mathrm{mag})\end{array}$ & $\begin{array}{c}\sigma_{22} \mu \mathrm{m} \\
(\mathrm{mag})\end{array}$ \\
\hline \multicolumn{9}{|c|}{ RSGC1 } \\
\hline F01 & 3.37 & 0.25 & 2.49 & 0.16 & -0.71 & 0.16 & -2.10 & 0.02 \\
\hline F02 & 3.88 & 0.09 & $\ldots$ & $\ldots$ & -1.22 & 0.07 & -2.77 & 0.01 \\
\hline F03 & 4.93 & 0.13 & 4.20 & 0.11 & 1.50 & 0.07 & -0.50 & 0.07 \\
\hline F04 & 5.54 & 0.08 & 4.72 & 0.05 & 1.27 & 0.06 & -0.83 & 0.02 \\
\hline F05 & 3.89 & 0.12 & 4.05 & 0.02 & 0.99 & 0.07 & -0.36 & 0.02 \\
\hline F06 & 5.41 & 0.06 & 4.42 & 0.04 & 2.24 & 0.02 & -0.17 & 0.02 \\
\hline F07 & 5.57 & 0.11 & 5.26 & 0.45 & 3.78 & 0.42 & $4.89 \dagger$ & $\ldots$ \\
\hline F08 & 3.89 & 0.12 & 4.05 & 0.02 & 0.99 & 0.07 & -0.36 & 0.02 \\
\hline F09 & $\ldots$ & $\ldots$ & $\ldots$ & $\ldots$ & $\ldots$ & $\ldots$ & $\ldots$ & $\ldots$ \\
\hline F10 & 4.68 & 0.09 & 3.86 & 0.06 & 2.14 & 0.03 & -0.04 & 0.02 \\
\hline F11 & 5.49 & 0.06 & 4.42 & 0.05 & 2.50 & 0.03 & 0.82 & 0.04 \\
\hline F12 & 4.92 & 0.06 & 3.97 & 0.04 & 2.55 & 0.02 & 1.31 & 0.02 \\
\hline F13 & 3.47 & 0.16 & 2.02 & 0.08 & $\ldots$ & $\ldots$ & -1.98 & 0.01 \\
\hline F14 & 5.15 & 0.05 & 4.63 & 0.04 & 3.43 & 0.02 & 2.26 & 0.03 \\
\hline \multicolumn{9}{|c|}{ RSGC2 } \\
\hline St2-01 & 4.96 & 0.07 & 4.22 & 0.05 & 2.98 & 0.02 & 1.56 & 0.03 \\
\hline St2-02 & 4.81 & 0.07 & 4.22 & 0.04 & 3.14 & 0.03 & 1.61 & 0.03 \\
\hline St2-03 & 2.85 & 0.18 & 1.93 & 0.11 & -0.26 & 0.03 & -1.26 & 0.02 \\
\hline St2-04 & 4.51 & 0.09 & 3.91 & 0.05 & 1.81 & 0.02 & 0.55 & 0.03 \\
\hline St2-05 & 4.62 & 0.08 & 4.11 & 0.05 & 2.85 & 0.02 & 1.61 & 0.03 \\
\hline \multicolumn{9}{|c|}{ RSGC2 SW } \\
\hline St2-08 & 4.82 & 0.06 & 4.19 & 0.05 & 3.12 & 0.02 & 1.45 & 0.09 \\
\hline St2-11 & 6.33 & 0.05 & 4.07 & 0.06 & 1.33 & 0.07 & -0.63 & 0.03 \\
\hline St2-14 & 5.00 & 0.05 & 4.37 & 0.04 & 2.27 & 0.02 & 0.66 & 0.04 \\
\hline St2-18 & 2.28 & 0.25 & 1.44 & 0.01 & -1.27 & 0.06 & -2.12 & 0.02 \\
\hline St2-26 & 5.72 & 0.05 & 4.69 & 0.04 & 3.25 & 0.02 & 1.76 & 0.04 \\
\hline \multicolumn{9}{|c|}{ Per OB1 } \\
\hline W Per & 2.56 & 0.03 & 1.70 & 0.01 & -1.73 & 0.21 & -2.89 & 0.001 \\
\hline S Per & $\ldots$ & $\ldots$ & $\ldots$ & $\ldots$ & -3.11 & 0.29 & -4.32 & 0.001 \\
\hline T Per & 2.43 & 0.12 & 1.85 & 0.07 & 1.01 & 0.01 & -0.19 & 0.01 \\
\hline V605 Cas & 2.21 & 0.32 & 1.58 & 0.09 & 0.71 & 0.02 & -0.44 & 0.02 \\
\hline V778 Cas & 2.33 & 0.06 & 1.94 & 0.01 & 0.70 & 0.01 & 0.32 & 0.01 \\
\hline PR Per & 2.01 & 0.29 & 1.52 & 0.04 & 0.49 & 0.01 & -0.56 & 0.02 \\
\hline & & & & 14 & & & & \\
\hline
\end{tabular}


Table 12

(Continued)

\begin{tabular}{lccccccrc}
\hline \hline Source & \multicolumn{7}{c}{ WISE $^{\mathrm{a}}$} \\
\cline { 2 - 9 } Name & $\begin{array}{c}3.4 \mu \mathrm{m} \\
(\mathrm{mag})\end{array}$ & $\begin{array}{c}\sigma_{3.4 \mu \mathrm{m}} \\
(\mathrm{mag})\end{array}$ & $\begin{array}{c}4.6 \mu \mathrm{m} \\
(\mathrm{mag})\end{array}$ & $\begin{array}{c}\sigma_{4.6 \mu \mathrm{m}} \\
(\mathrm{mag})\end{array}$ & $\begin{array}{r}12 \mu \mathrm{m} \\
(\mathrm{mag})\end{array}$ & $\begin{array}{r}\sigma_{12 \mu \mathrm{m}} \\
(\mathrm{mag})\end{array}$ & $\begin{array}{r}22 \mu \mathrm{m} \\
(\mathrm{mag})\end{array}$ & $\begin{array}{c}\sigma_{22} \mu \mathrm{m} \\
(\mathrm{mag})\end{array}$ \\
\hline FZ Per & 2.27 & 0.16 & 1.49 & 0.12 & 0.62 & 0.01 & 0.07 & 0.01 \\
BD+59 372 & 3.19 & 0.13 & 2.62 & 0.05 & 2.69 & 0.01 & 2.60 & 0.02 \\
XX Per & $\ldots$ & $\ldots$ & 1.41 & 0.05 & -0.94 & 0.04 & -1.86 & 0.01 \\
HD 236947 & 2.66 & 0.12 & 1.86 & 0.09 & 1.99 & 0.01 & 1.68 & 0.02 \\
KK Per & 2.12 & 0.27 & 1.68 & 0.07 & -0.27 & 0.01 & -0.79 & 0.01 \\
V550 Per & 2.94 & 0.14 & 2.41 & 0.09 & 1.90 & 0.01 & 1.33 & 0.01 \\
PP Per & 2.60 & 0.26 & 2.08 & 0.12 & 2.01 & 0.02 & 1.64 & 0.03 \\
BU Per & 1.94 & 0.48 & 1.30 & 0.20 & -0.64 & 0.02 & -1.99 & 0.004 \\
AD Per & 1.88 & 0.47 & 1.30 & 0.19 & -0.17 & 0.02 & -0.91 & 0.01 \\
SU Per & $\ldots$ & $\ldots$ & 1.38 & 0.21 & -1.29 & 0.02 & -1.88 & 0.01 \\
RS Per & $\ldots$ & $\ldots$ & $\ldots$ & $\ldots$ & -1.23 & 0.03 & -2.43 & 0.01 \\
V439 Per & 2.56 & 0.26 & 2.03 & 0.07 & 1.13 & 0.01 & 1.01 & 0.01 \\
V403 Per & $\ldots$ & $\ldots$ & $\ldots$ & $\ldots$ & 1.94 & 0.02 & 1.78 & 0.02 \\
V441 Per & $\ldots$ & $\ldots$ & 1.33 & 0.05 & 0.06 & 0.01 & -0.95 & 0.01 \\
YZ Per & 2.26 & 0.01 & 1.81 & 0.02 & -1.03 & 0.01 & -1.72 & 0.01 \\
GP Cas & $\ldots$ & $\ldots$ & $\ldots$ & $\ldots$ & -0.34 & 0.02 & -1.30 & 0.01 \\
V648 Cas & 2.02 & 0.34 & $\ldots$ & $\ldots$ & -0.70 & 0.01 & -1.79 & 0.004 \\
\hline & & & & Mc8 & & & & \\
\hline Mc8-01 & 3.95 & 0.08 & 2.83 & 0.06 & 1.52 & 0.02 & 0.48 & 0.02 \\
Mc8-02 & 5.14 & 0.06 & 4.39 & 0.04 & 3.31 & 0.02 & 2.28 & 0.03 \\
Mc8-03 & 5.53 & 0.06 & 4.64 & 0.03 & 2.82 & 0.02 & 1.31 & 0.02 \\
Mc8-04 & 4.84 & 0.07 & 3.24 & 0.05 & 1.50 & 0.02 & 0.49 & 0.03 \\
Mc8-05 & 5.31 & 0.06 & 4.16 & 0.04 & 2.57 & 0.02 & 1.31 & 0.03 \\
Mc8-06 & 3.62 & 0.09 & 3.29 & 0.05 & 2.35 & 0.02 & 0.73 & 0.02 \\
\hline
\end{tabular}

Notes. ${ }^{\text {a }}$ For saturating sources, the measured fluxes are calibrated by non-saturated wings of their profiles.

$\dagger$ The dagger represents 95\% confidence upper limit.

Table 13

Infrared Photometric Data 3

\begin{tabular}{|c|c|c|c|c|c|c|c|c|}
\hline \multirow{2}{*}{$\begin{array}{l}\text { Source } \\
\text { Name }\end{array}$} & \multicolumn{8}{|c|}{ GLIMPSE $^{\mathrm{a}}$} \\
\hline & $\begin{array}{c}3.6 \mu \mathrm{m} \\
(\mathrm{mag})\end{array}$ & $\begin{array}{c}\sigma_{3.6 \mu \mathrm{m}} \\
(\mathrm{mag})\end{array}$ & $\begin{array}{c}4.5 \mu \mathrm{m} \\
(\mathrm{mag})\end{array}$ & $\begin{array}{c}\sigma_{4.5 \mu \mathrm{m}} \\
(\mathrm{mag})\end{array}$ & $\begin{array}{c}5.8 \mu \mathrm{m} \\
(\mathrm{mag})\end{array}$ & $\begin{array}{c}\sigma_{5.8 \mu \mathrm{m}}(\mathrm{mag})\end{array}$ & $\begin{array}{c}8.0 \mu \mathrm{m} \\
(\mathrm{mag})\end{array}$ & $\begin{array}{c}\sigma_{8.0 \mu \mathrm{m}} \\
(\mathrm{mag})\end{array}$ \\
\hline \multicolumn{9}{|c|}{ RSGC1 } \\
\hline F01 & $\ldots$ & $\ldots$ & $\ldots$ & $\ldots$ & $\ldots$ & $\ldots$ & $\ldots$ & $\ldots$ \\
\hline F02 & $\ldots$ & $\ldots$ & 3.54 & 0.07 & 2.69 & 0.05 & $\ldots$ & $\ldots$ \\
\hline F03 & 4.23 & 0.11 & $\ldots$ & $\ldots$ & 3.64 & 0.04 & $\ldots$ & $\ldots$ \\
\hline F04 & 4.21 & 0.04 & 4.13 & 0.05 & 3.60 & 0.04 & $\ldots$ & $\ldots$ \\
\hline F05 & 5.24 & 0.22 & $\ldots$ & $\ldots$ & 3.85 & 0.03 & 5.05 & 0.24 \\
\hline F06 & $\ldots$ & $\ldots$ & $\ldots$ & $\ldots$ & 3.83 & 0.03 & $\ldots$ & $\ldots$ \\
\hline F07 & $\ldots$ & $\ldots$ & 4.62 & 0.07 & 3.86 & 0.03 & 3.25 & 0.04 \\
\hline F08 & $\ldots$ & $\ldots$ & $\ldots$ & 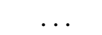 & 3.89 & 0.03 & $\ldots$ & $\ldots$ \\
\hline F09 & 4.47 & 0.05 & 4.55 & 0.07 & 3.80 & 0.03 & $\ldots$ & $\ldots$ \\
\hline F10 & 4.70 & 0.03 & 4.64 & 0.04 & 3.96 & 0.03 & $\ldots$ & $\ldots$ \\
\hline F11 & 4.43 & 0.10 & $\ldots$ & $\ldots$ & 3.89 & 0.03 & $\ldots$ & $\ldots$ \\
\hline F12 & $\ldots$ & $\ldots$ & $\ldots$ & $\ldots$ & 4.17 & 0.03 & 3.99 & 0.04 \\
\hline F13 & $\ldots$ & $\ldots$ & $\ldots$ & $\ldots$ & $\ldots$ & $\ldots$ & $\ldots$ & $\ldots$ \\
\hline F14 & 5.89 & 0.12 & 5.88 & 0.12 & 4.65 & 0.03 & 4.36 & 0.03 \\
\hline \multicolumn{9}{|c|}{ RSGC2 } \\
\hline St2-01 & 4.65 & 0.08 & 4.74 & 0.07 & 4.34 & 0.03 & 4.26 & 0.03 \\
\hline St2-02 & 4.56 & 0.05 & $\ldots$ & $\ldots$ & 4.23 & 0.03 & 4.11 & 0.03 \\
\hline St2-03 & $\ldots$ & $\ldots$ & $\ldots$ & $\ldots$ & $\ldots$ & $\ldots$ & $\ldots$ & $\ldots$ \\
\hline St2-04 & $\ldots$ & $\ldots$ & $\ldots$ & $\ldots$ & $\ldots$ & $\ldots$ & $\ldots$ & $\ldots$ \\
\hline St2-05 & 4.72 & 0.19 & $\ldots$ & $\ldots$ & 4.23 & 0.03 & 4.02 & 0.03 \\
\hline \multicolumn{9}{|c|}{ RSGC2 SW } \\
\hline St2-08 & 4.56 & 0.06 & $\ldots$ & $\ldots$ & 4.22 & 0.02 & 4.22 & 0.03 \\
\hline St2-11 & $\ldots$ & $\ldots$ & $\ldots$ & $\ldots$ & $\ldots$ & $\ldots$ & $\ldots$ & $\ldots$ \\
\hline St2-14 & 3.89 & 0.08 & 3.98 & 0.05 & 3.61 & 0.03 & $\ldots$ & $\ldots$ \\
\hline St2-18 & $\ldots$ & $\ldots$ & $\ldots$ & $\ldots$ & $\ldots$ & $\ldots$ & $\ldots$ & $\ldots$ \\
\hline St2-26 & $\ldots$ & $\ldots$ & $\ldots$ & $\ldots$ & $\ldots$ & $\ldots$ & $\ldots$ & $\ldots$ \\
\hline
\end{tabular}


Table 13

(Continued)

\begin{tabular}{lcccccccc}
\hline \hline Source & \multicolumn{7}{c}{ GLIMPSE $^{\mathrm{a}}$} \\
\cline { 2 - 9 } Name & $\begin{array}{c}3.6 \mu \mathrm{m} \\
(\mathrm{mag})\end{array}$ & $\begin{array}{c}\sigma_{3.6 \mu \mathrm{m}} \\
(\mathrm{mag})\end{array}$ & $\begin{array}{c}4.5 \mu \mathrm{m} \\
(\mathrm{mag})\end{array}$ & $\begin{array}{c}\sigma_{4.5 \mu \mathrm{m}} \\
(\mathrm{mag})\end{array}$ & $\begin{array}{c}5.8 \mu \mathrm{m} \\
(\mathrm{mag})\end{array}$ & $\begin{array}{c}\sigma_{5.8 \mu \mathrm{m}} \\
(\mathrm{mag})\end{array}$ & $\begin{array}{c}8.0 \mu \mathrm{m} \\
(\mathrm{mag})\end{array}$ & $\begin{array}{c}\sigma_{8.0} \mu \mathrm{m} \\
(\mathrm{mag})\end{array}$ \\
\hline & & & \multicolumn{7}{c}{$\mathrm{Mc8}$} \\
\hline Mc8-01 & 4.02 & 0.21 & 4.95 & 0.24 & 3.30 & 0.04 & $\ldots$ & $\ldots$ \\
Mc8-02 & 4.65 & 0.06 & $\ldots$ & $\ldots$ & 4.27 & 0.03 & 4.21 & 0.02 \\
Mc8-03 & $\ldots$ & $\ldots$ & 5.91 & 0.17 & 4.46 & 0.03 & 3.98 & 0.02 \\
Mc8-04 & 4.43 & 0.14 & $\ldots$ & $\ldots$ & 3.58 & 0.03 & 3.27 & 0.04 \\
Mc8-05 & 5.03 & 0.26 & 4.85 & 0.07 & 4.35 & 0.03 & 4.22 & 0.03 \\
Mc8-06 & 3.99 & 0.06 & 4.31 & 0.05 & 3.83 & 0.03 & 3.54 & 0.03 \\
\hline
\end{tabular}

Note. ${ }^{a}$ For saturating sources, the measured fluxes are calibrated by non-saturated wings of their profiles. The aperture corrections of point sources were made using the effective correction factors given by Reach et al. (2005).

Table 14

Infrared Photometric Data 4

\begin{tabular}{|c|c|c|c|c|c|c|c|c|c|c|c|c|}
\hline \multirow{2}{*}{$\begin{array}{l}\text { Source } \\
\text { Name }\end{array}$} & \multicolumn{12}{|c|}{$M S X$} \\
\hline & $\begin{array}{c}4.29 \mu \mathrm{m} \\
(\mathrm{Jy})\end{array}$ & $\begin{array}{c}\sigma_{4.29 \mu \mathrm{m}} \\
(\mathrm{Jy})\end{array}$ & $\begin{array}{c}4.35 \mu \mathrm{m} \\
(\mathrm{Jy})\end{array}$ & $\begin{array}{c}\sigma_{4.35 \mu \mathrm{m}} \\
(\mathrm{Jy})\end{array}$ & $\begin{array}{c}8.28 \mu \mathrm{m} \\
(\mathrm{Jy})\end{array}$ & $\begin{array}{c}\sigma_{8.28 \mu \mathrm{m}} \\
(\mathrm{Jy})\end{array}$ & $\begin{array}{c}12.13 \mu \mathrm{m} \\
(\mathrm{Jy})\end{array}$ & $\begin{array}{c}\sigma_{12.13 \mu \mathrm{m}} \\
(\mathrm{Jy})\end{array}$ & $\begin{array}{c}14.65 \mu \mathrm{m} \\
(\mathrm{Jy})\end{array}$ & $\begin{array}{c}\sigma_{14.65 \mu \mathrm{m}} \\
(\mathrm{Jy})\end{array}$ & $\begin{array}{c}21.34 \mu \mathrm{m} \\
(\mathrm{Jy})\end{array}$ & $\begin{array}{c}\sigma_{21.34 \mu \mathrm{m}} \\
(\mathrm{Jy})\end{array}$ \\
\hline \multicolumn{13}{|c|}{ Wd1 } \\
\hline W 237 & 43.40 & 4.21 & 45.50 & 4.19 & 61.17 & 2.51 & 100.60 & 5.03 & 82.95 & 5.06 & 101.39 & 6.08 \\
\hline W 26 & 143.27 & 12.18 & 171.67 & 15.11 & 216.37 & 8.87 & 326.91 & 16.35 & 254.14 & 15.50 & 240.82 & 14.45 \\
\hline W 20 & 30.33 & 3.25 & 37.82 & 3.56 & 37.06 & 1.52 & 57.67 & 2.88 & 52.18 & 3.18 & 62.56 & 3.75 \\
\hline W 75 & $\ldots$ & $\ldots$ & 19.05 & 2.04 & 13.27 & 0.54 & 14.43 & 0.72 & 11.25 & 0.69 & 11.35 & 0.68 \\
\hline \multicolumn{13}{|c|}{ RSGC1 } \\
\hline F01 & $\ldots$ & $\ldots$ & $\ldots$ & $\ldots$ & $\ldots$ & $\ldots$ & $\ldots$ & $\ldots$ & $\ldots$ & $\ldots$ & $\ldots$ & $\ldots$ \\
\hline F02 & $\ldots$ & $\ldots$ & $\ldots$ & $\ldots$ & $\ldots$ & $\ldots$ & $\ldots$ & $\ldots$ & $\ldots$ & $\ldots$ & $\ldots$ & $\ldots$ \\
\hline F03 & $\ldots$ & $\ldots$ & 6.19 & 0.59 & 5.77 & 0.24 & 11.91 & 0.60 & 9.68 & 0.59 & 9.82 & 0.59 \\
\hline F04 & $\ldots$ & $\ldots$ & 5.96 & 0.57 & 5.42 & 0.22 & 10.71 & 0.54 & 8.75 & 0.53 & 6.99 & 0.42 \\
\hline F05 & $\ldots$ & $\ldots$ & $\ldots$ & $\ldots$ & $\ldots$ & $\ldots$ & $\ldots$ & $\ldots$ & $\ldots$ & $\ldots$ & $\ldots$ & $\ldots$ \\
\hline F06 & $\ldots$ & $\ldots$ & 2.98 & 0.34 & 3.21 & 0.13 & 4.98 & 0.25 & 3.45 & 0.21 & 3.19 & 0.19 \\
\hline F07 & $\ldots$ & $\ldots$ & $\ldots$ & $\ldots$ & $\ldots$ & $\ldots$ & $\ldots$ & $\ldots$ & $\ldots$ & $\ldots$ & $\ldots$ & $\ldots$ \\
\hline F08 & $\ldots$ & $\ldots$ & $\ldots$ & $\ldots$ & $\ldots$ & $\ldots$ & $\ldots$ & $\ldots$ & $\ldots$ & $\ldots$ & $\ldots$ & $\ldots$ \\
\hline F09 & $\ldots$ & $\ldots$ & 5.90 & 0.57 & 5.14 & 0.21 & 7.82 & 0.39 & 6.70 & 0.41 & 8.74 & 0.52 \\
\hline F10 & $\ldots$ & $\ldots$ & $\ldots$ & $\ldots$ & $\ldots$ & $\ldots$ & $\ldots$ & $\ldots$ & $\ldots$ & $\ldots$ & $\ldots$ & $\ldots$ \\
\hline F11 & $\ldots$ & $\ldots$ & 3.49 & 0.38 & 2.66 & 0.11 & 3.82 & 0.19 & 2.79 & 0.17 & 1.77 & 0.11 \\
\hline F12 & $\ldots$ & $\ldots$ & 3.47 & 0.38 & 1.87 & 0.08 & 2.49 & 0.12 & 1.44 & 0.09 & 1.15 & 0.07 \\
\hline F13 & 16.28: & 7.65 & 8.69 & 0.81 & 8.71 & 0.36 & 14.17 & 0.71 & 12.30 & 0.75 & 14.17 & 0.85 \\
\hline F14 & $\ldots$ & $\ldots$ & 2.28: & 0.30 & 0.97 & 0.04 & 0.84 & 0.05 & 0.64 & 0.04 & 0.41: & 0.03 \\
\hline \multicolumn{13}{|c|}{ RSGC2 } \\
\hline St2-01 & $\ldots$ & $\ldots$ & $\ldots$ & $\ldots$ & 2.03 & 0.08 & 1.93 & 0.11 & 1.21 & 0.08 & $\ldots$ & $\ldots$ \\
\hline St2-02 & $\ldots$ & $\ldots$ & $\ldots$ & $\ldots$ & 1.63 & 0.07 & 1.48 & 0.09 & $0.67:$ & 0.06 & $\ldots$ & $\ldots$ \\
\hline St2-03 & $\ldots$ & $\ldots$ & 15.74: & 2.27 & 13.14 & 0.54 & 19.91 & 1.00 & 15.38 & 0.94 & 13.19 & 0.79 \\
\hline St2-04 & 18.16: & 9.03 & 10.07: & 1.54 & 5.12 & 0.21 & 6.27 & 0.31 & 4.02 & 0.25 & 2.49 & 0.17 \\
\hline St2-05 & $\ldots$ & $\ldots$ & $\ldots$ & $\ldots$ & 1.71 & 0.07 & 1.52 & 0.10 & 1.08 & 0.08 & $\ldots$ & $\ldots$ \\
\hline \multicolumn{13}{|c|}{ RSGC2 SW } \\
\hline St2-08 & $\ldots$ & $\ldots$ & $\ldots$ & $\ldots$ & 1.38 & 0.06 & 1.54 & 0.10 & 1.01 & 0.07 & $\ldots$ & $\ldots$ \\
\hline St2-11 & $\ldots$ & $\ldots$ & $\cdots$ & $\ldots$ & 7.35 & 0.30 & 16.61 & 0.83 & 16.56 & 1.01 & 14.25 & 0.86 \\
\hline St2-14 & $\ldots$ & $\ldots$ & $\ldots$ & $\ldots$ & 3.38 & 0.14 & 4.88 & 0.25 & 3.92 & 0.24 & 2.94 & 0.19 \\
\hline St2-18 & 27.77 & 2.83 & 48.62 & 5.01 & 31.24 & 1.28 & 56.86 & 2.84 & 45.89 & 2.80 & 35.74 & 2.14 \\
\hline St2-26 & $\ldots$ & $\cdots$ & $\ldots$ & $\cdots$ & 1.37 & 0.06 & 1.83 & 0.11 & 1.34 & 0.09 & $\ldots$ & $\ldots$ \\
\hline \multicolumn{13}{|c|}{ Per OB1 } \\
\hline W Per & 50.99 & 5.15 & 63.11 & 5.87 & 63.45 & 2.60 & 79.47 & 3.97 & 62.61 & 3.82 & 68.76 & 4.13 \\
\hline S Per & 147.27 & 12.81 & 186.16 & 16.57 & 162.82 & 6.68 & 274.56 & 13.73 & 185.62 & 11.32 & 178.67 & 10.72 \\
\hline T Per & 29.18: & 3.73 & 19.93 & 2.13 & 10.57 & 0.43 & 8.66 & 0.43 & 6.57 & 0.40 & 4.75 & 0.30 \\
\hline V605 Cas & 16.99: & 2.46 & 27.32 & 2.71 & 12.54 & 0.51 & 11.32 & 0.57 & 7.69 & 0.47 & 7.93 & 0.48 \\
\hline V778 Cas & $\ldots$ & $\ldots$ & 32.23 & 3.35 & 10.22 & 0.42 & 7.20 & 0.38 & 4.94 & 0.32 & 2.89: & 0.51 \\
\hline PR Per & 26.75: & 3.56 & 28.78: & 3.28 & 14.36 & 0.59 & 11.78 & 0.59 & 8.90 & 0.54 & 8.26 & 0.51 \\
\hline FZ Per & $\ldots$ & $\ldots$ & $\ldots$ & $\ldots$ & 12.19 & 0.50 & 10.90 & 0.54 & 6.52 & 0.40 & 4.13 & 0.27 \\
\hline BD+59 372 & $\ldots$ & $\ldots$ & $\ldots$ & $\ldots$ & 2.80 & 0.11 & 1.57 & 0.10 & 0.89: & 0.07 & $\ldots$ & $\ldots$ \\
\hline XX Per & $\cdots$ & $\ldots$ & $\cdots$ & $\ldots$ & $\ldots$ & $\ldots$ & $\ldots$ & $\ldots$ & $\ldots$ & $\cdots$ & $\cdots$ & $\ldots$ \\
\hline HD 236947 & $\ldots$ & $\ldots$ & $\ldots$ & $\ldots$ & $\ldots$ & $\ldots$ & $\ldots$ & $\ldots$ & $\ldots$ & $\ldots$ & $\ldots$ & $\ldots$ \\
\hline
\end{tabular}


Table 14

(Continued)

\begin{tabular}{|c|c|c|c|c|c|c|c|c|c|c|c|c|}
\hline \multirow{2}{*}{$\begin{array}{l}\text { Source } \\
\text { Name }\end{array}$} & \multicolumn{12}{|c|}{$M S X$} \\
\hline & $\begin{array}{l}4.29 \mu \mathrm{m} \\
(\mathrm{Jy})\end{array}$ & $\begin{array}{c}\sigma_{4.29 \mu \mathrm{m}} \\
(\mathrm{Jy})\end{array}$ & $\begin{array}{c}4.35 \mu \mathrm{m} \\
(\mathrm{Jy})\end{array}$ & $\begin{array}{c}\sigma_{4.35 \mu \mathrm{m}} \\
(\mathrm{Jy})\end{array}$ & $\begin{array}{c}8.28 \mu \mathrm{m} \\
(\mathrm{Jy})\end{array}$ & $\begin{array}{c}\sigma_{8.28 \mu \mathrm{m}} \\
(\mathrm{Jy})\end{array}$ & $\begin{array}{l}12.13 \mu \mathrm{m} \\
(\mathrm{Jy})\end{array}$ & $\begin{array}{c}\sigma_{12.13 \mu \mathrm{m}} \\
(\mathrm{Jy})\end{array}$ & $\begin{array}{c}14.65 \mu \mathrm{m} \\
(\mathrm{Jy})\end{array}$ & $\begin{array}{c}\sigma_{14.65 \mu \mathrm{m}} \\
(\mathrm{Jy})\end{array}$ & $\begin{array}{c}21.34 \mu \mathrm{m} \\
(\mathrm{Jy})\end{array}$ & $\begin{array}{c}\sigma_{21.34 \mu \mathrm{m}} \\
(\mathrm{Jy})\end{array}$ \\
\hline KK Per & $\ldots$ & $\ldots$ & 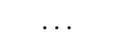 & 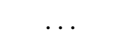 & . & $\ldots$ & $\ldots$ & $\ldots$ & $\ldots$ & $\ldots$ & $\ldots$ & $\ldots$ \\
\hline V550 Per & $\ldots$ & $\ldots$ & 16.67: & 2.32 & 5.19 & 0.21 & 3.51 & 0.22 & 1.81 & 0.15 & $\ldots$ & $\ldots$ \\
\hline PP Per & 32.92: & 9.81 & 12.56: & 2.10 & 5.48 & 0.22 & 2.74 & 0.17 & 2.31 & 0.16 & $\ldots$ & $\ldots$ \\
\hline BU Per & 33.14 & 3.38 & 34.91 & 3.56 & 33.10 & 1.36 & 36.70 & 1.83 & 26.30 & 1.60 & 30.21 & 1.81 \\
\hline AD Per & $\ldots$ & $\ldots$ & 35.80 & 3.37 & 20.75 & 0.85 & 20.44 & 1.02 & 13.12 & 0.80 & 11.25 & 0.69 \\
\hline SU Per & $\ldots$ & $\ldots$ & 20.58 & 2.41 & 43.77 & 1.79 & 40.03 & 2.00 & 24.11 & 1.47 & 30.08 & 1.80 \\
\hline RS Per & $\ldots$ & $\ldots$ & 73.08 & 6.72 & 57.59 & 2.36 & 59.47 & 2.97 & 42.78 & 2.61 & 41.09 & 2.47 \\
\hline V439 Per & $\ldots$ & $\ldots$ & 17.79: & 2.38 & 7.33 & 0.30 & 5.18 & 0.27 & 3.20 & 0.20 & 2.39 & 0.17 \\
\hline V403 Per & 30.91 & 3.21 & 15.85: & 2.25 & 5.31 & 0.22 & 2.71 & 0.16 & 1.83 & 0.12 & $\ldots$ & $\ldots$ \\
\hline V441 Per & 39.42 & 3.86 & 44.46 & 4.31 & 19.32 & 0.79 & 16.81 & 0.84 & 13.26 & 0.81 & 11.10 & 0.68 \\
\hline YZ Per & 37.91 & 4.25 & 42.70 & 4.18 & 33.62 & 1.38 & 31.78 & 1.59 & 21.23 & 1.29 & 20.18 & 1.21 \\
\hline GP Cas & 45.68 & 4.29 & 48.57 & 4.57 & 22.97 & 0.94 & 23.51 & 1.18 & 17.14 & 1.05 & 16.10 & 0.97 \\
\hline V648 Cas & 54.72 & 5.03 & 50.40 & 4.74 & 31.87 & 1.31 & 34.81 & 1.74 & 26.89 & 1.64 & 24.57 & 1.47 \\
\hline \multicolumn{13}{|c|}{ Mc8 } \\
\hline Mc8-01 & 3.80: & 0.48 & $\ldots$ & $\ldots$ & 4.66 & 0.19 & 5.61 & 0.28 & 4.68 & 0.29 & 3.67 & 0.22 \\
\hline Mc8-02 & 2.82: & 0.42 & $\ldots$ & $\ldots$ & 1.28 & 0.05 & 1.04 & 0.05 & 0.79 & 0.05 & 0.73 & 0.05 \\
\hline Mc8-03 & 10.78: & 2.12 & $\ldots$ & $\ldots$ & 3.39 & 0.14 & 4.12 & 0.21 & 3.12 & 0.19 & 3.24 & 0.19 \\
\hline Mc8-04 & 3.23: & 0.45 & $\ldots$ & $\ldots$ & 3.21 & 0.13 & 3.64 & 0.18 & 2.80 & 0.17 & 2.04 & 0.12 \\
\hline Mc8-05 & $\ldots$ & $\ldots$ & $\ldots$ & $\ldots$ & 1.44 & 0.06 & 2.17 & 0.11 & 1.63 & 0.10 & 1.02 & 0.07 \\
\hline Mc8-06 & 5.00 & 0.55 & $\ldots$ & $\ldots$ & 2.46 & 0.10 & 2.27 & 0.11 & 1.45 & 0.09 & 0.75 & 0.05 \\
\hline
\end{tabular}

Note. The colon represents uncertain detection.

Table 15

Infrared Photometric Data 5

\begin{tabular}{|c|c|c|c|c|}
\hline \multirow{2}{*}{$\begin{array}{l}\text { Source } \\
\text { Name }\end{array}$} & \multicolumn{4}{|c|}{ AKARI } \\
\hline & $\begin{array}{c}9.0 \mu \mathrm{m} \\
(\mathrm{Jy})\end{array}$ & $\begin{array}{c}\sigma_{9 \mu \mathrm{m}} \\
(\mathrm{Jy})\end{array}$ & $\begin{array}{c}18.0 \mu \mathrm{m} \\
(\mathrm{Jy})\end{array}$ & $\underset{(\mathrm{Jy})}{\sigma_{18 \mu \mathrm{m}}}$ \\
\hline \multicolumn{5}{|c|}{ Wd1 } \\
\hline W 237 & 91.65 & 7.35 & 113.80 & 0.67 \\
\hline W 26 & 296.50 & 7.68 & 449.70 & 4.31 \\
\hline W 20 & 61.41 & 0.28 & 111.70 & 0.67 \\
\hline W 75 & 24.26 & 1.64 & $\ldots$ & $\ldots$ \\
\hline \multicolumn{5}{|c|}{ RSGC1 } \\
\hline F01 & 11.47 & 0.34 & $\ldots$ & $\ldots$ \\
\hline F02 & 12.69 & 0.17 & $\ldots$ & $\ldots$ \\
\hline F03 & 6.46 & 0.54 & $\ldots$ & $\ldots$ \\
\hline F04 & 5.75 & 0.03 & $\ldots$ & $\ldots$ \\
\hline F05 & 7.86 & 0.45 & $\ldots$ & $\ldots$ \\
\hline F06 & 5.17 & 0.58 & $\ldots$ & $\ldots$ \\
\hline F07 & $\ldots$ & $\ldots$ & $\ldots$ & $\ldots$ \\
\hline F08 & 7.86 & 0.45 & $\ldots$ & $\ldots$ \\
\hline F09 & $\ldots$ & $\ldots$ & $\ldots$ & $\ldots$ \\
\hline F10 & $\ldots$ & $\ldots$ & $\ldots$ & $\ldots$ \\
\hline F11 & 4.01 & 0.70 & $\ldots$ & $\ldots$ \\
\hline F12 & 2.16 & 0.12 & $\ldots$ & $\ldots$ \\
\hline F13 & 13.30 & 0.41 & $\ldots$ & $\ldots$ \\
\hline F14 & 0.91 & 0.01 & $\ldots$ & $\ldots$ \\
\hline \multicolumn{5}{|c|}{ RSGC2 } \\
\hline St2-01 & 2.64 & 0.13 & 1.52 & 0.08 \\
\hline St2-02 & 1.51 & 0.05 & 0.56 & 0.05 \\
\hline St2-03 & 14.16 & 0.26 & 14.24 & 0.17 \\
\hline St2-04 & 6.98 & 1.43 & 3.68 & 0.08 \\
\hline St2-05 & 1.45 & 0.01 & 0.66 & 0.09 \\
\hline \multicolumn{5}{|c|}{ RSGC2 SW } \\
\hline St2-08 & 1.30 & 0.16 & $\ldots$ & $\ldots$ \\
\hline St2-11 & 8.62 & 0.41 & 12.31 & 0.24 \\
\hline St2-14 & $\ldots$ & $\ldots$ & 2.80 & 0.14 \\
\hline St2-18 & 33.98 & 0.41 & 36.78 & 0.08 \\
\hline St2-26 & 1.45 & 0.20 & 1.17 & 0.09 \\
\hline
\end{tabular}

Table 15

(Continued)

\begin{tabular}{|c|c|c|c|c|}
\hline \multirow{2}{*}{$\begin{array}{l}\text { Source } \\
\text { Name }\end{array}$} & \multicolumn{4}{|c|}{ AKARI } \\
\hline & $\begin{array}{c}9.0 \mu \mathrm{m} \\
(\mathrm{Jy})\end{array}$ & $\underset{(\mathrm{Jy})}{\sigma_{9 \mu \mathrm{m}}}$ & $\begin{array}{c}18.0 \mu \mathrm{m} \\
(\mathrm{Jy})\end{array}$ & $\underset{(\mathrm{Jy})}{\sigma_{18 \mu \mathrm{m}}}$ \\
\hline \multicolumn{5}{|c|}{ Per OB1 } \\
\hline W Per & 77.36 & 0.82 & 73.29 & 0.77 \\
\hline S Per & 349.70 & 3.68 & 285.50 & 11.80 \\
\hline T Per & 9.80 & 0.12 & 5.77 & 0.09 \\
\hline V605 Cas & 12.00 & 0.14 & 7.64 & 0.17 \\
\hline V778 Cas & 9.61 & 0.01 & 3.85 & 0.04 \\
\hline PR Per & 13.01 & 0.08 & 8.09 & 0.10 \\
\hline FZ Per & 12.03 & 0.12 & 4.98 & 0.05 \\
\hline $\mathrm{BD}+59372$ & 2.52 & 0.02 & 0.66 & 0.02 \\
\hline XX Per & 64.90 & 1.57 & 28.47 & 0.40 \\
\hline HD 236947 & 4.69 & 0.02 & 1.35 & 0.02 \\
\hline KK Per & 19.18 & 0.08 & 9.50 & 0.08 \\
\hline V550 Per & 4.63 & 0.03 & 1.67 & 0.01 \\
\hline PP Per & 4.76 & 0.05 & 1.43 & 0.01 \\
\hline BU Per & 36.59 & 0.51 & 30.27 & 0.51 \\
\hline AD Per & 20.81 & 0.15 & 11.90 & 0.13 \\
\hline SU Per & 45.48 & 0.47 & 30.69 & 0.41 \\
\hline RS Per & 66.55 & 0.88 & 47.17 & 0.85 \\
\hline V439 Per & 6.93 & 0.04 & 2.29 & 0.02 \\
\hline V403 Per & 4.81 & 0.02 & 1.33 & 0.03 \\
\hline V441 Per & $\ldots$ & $\ldots$ & 11.91 & 0.15 \\
\hline YZ Per & 37.24 & 0.36 & 25.05 & 0.08 \\
\hline GP Cas & 24.89 & 0.40 & 15.95 & 0.04 \\
\hline V648 Cas & 31.81 & 1.29 & 24.37 & 0.30 \\
\hline \multicolumn{5}{|c|}{ Mc8 } \\
\hline Mc8-01 & 5.17 & 0.11 & $\ldots$ & $\ldots$ \\
\hline Mc8-02 & 1.43 & 0.05 & 0.67 & 0.05 \\
\hline Mc8-03 & $\ldots$ & $\ldots$ & 2.62 & 0.13 \\
\hline Mc8-04 & $\ldots$ & $\ldots$ & 3.00 & 0.58 \\
\hline Mc8-05 & $\ldots$ & $\ldots$ & 1.17 & 0.04 \\
\hline Mc8-06 & $\ldots$ & $\ldots$ & $\ldots$ & $\ldots$ \\
\hline
\end{tabular}



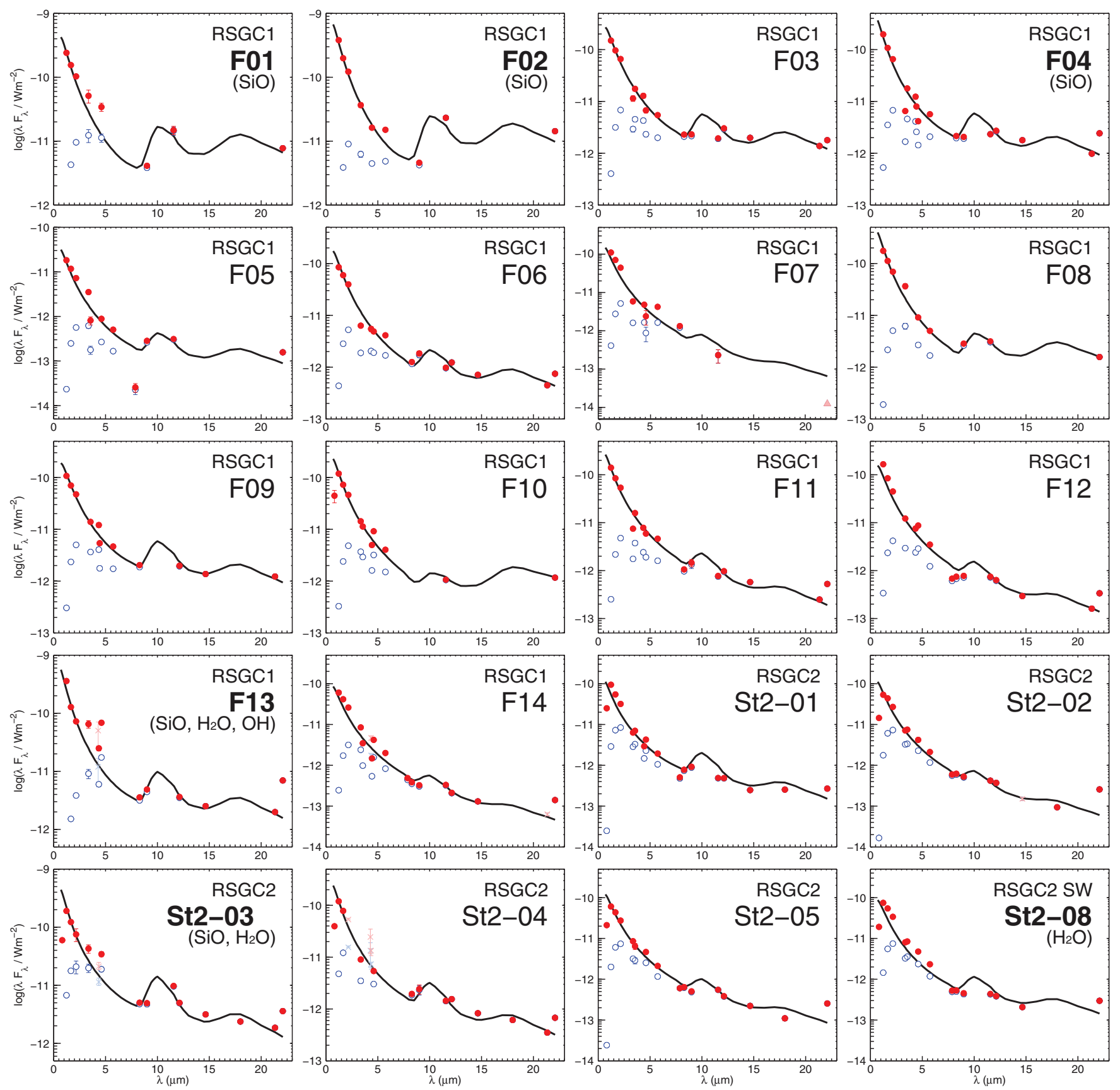

Figure 9. Spectral energy distributions of 53 known RSGs in five massive clusters. The notations of data points and model curves are the same as Figure 3. The names of parent clusters (small font) and object names (large font) are given in the upper right corners of each panel. The names of objects with maser detections are indicated in boldface, and detected maser molecules are presented in the parenthesis.

(A color version of this figure is available in the online journal.) 

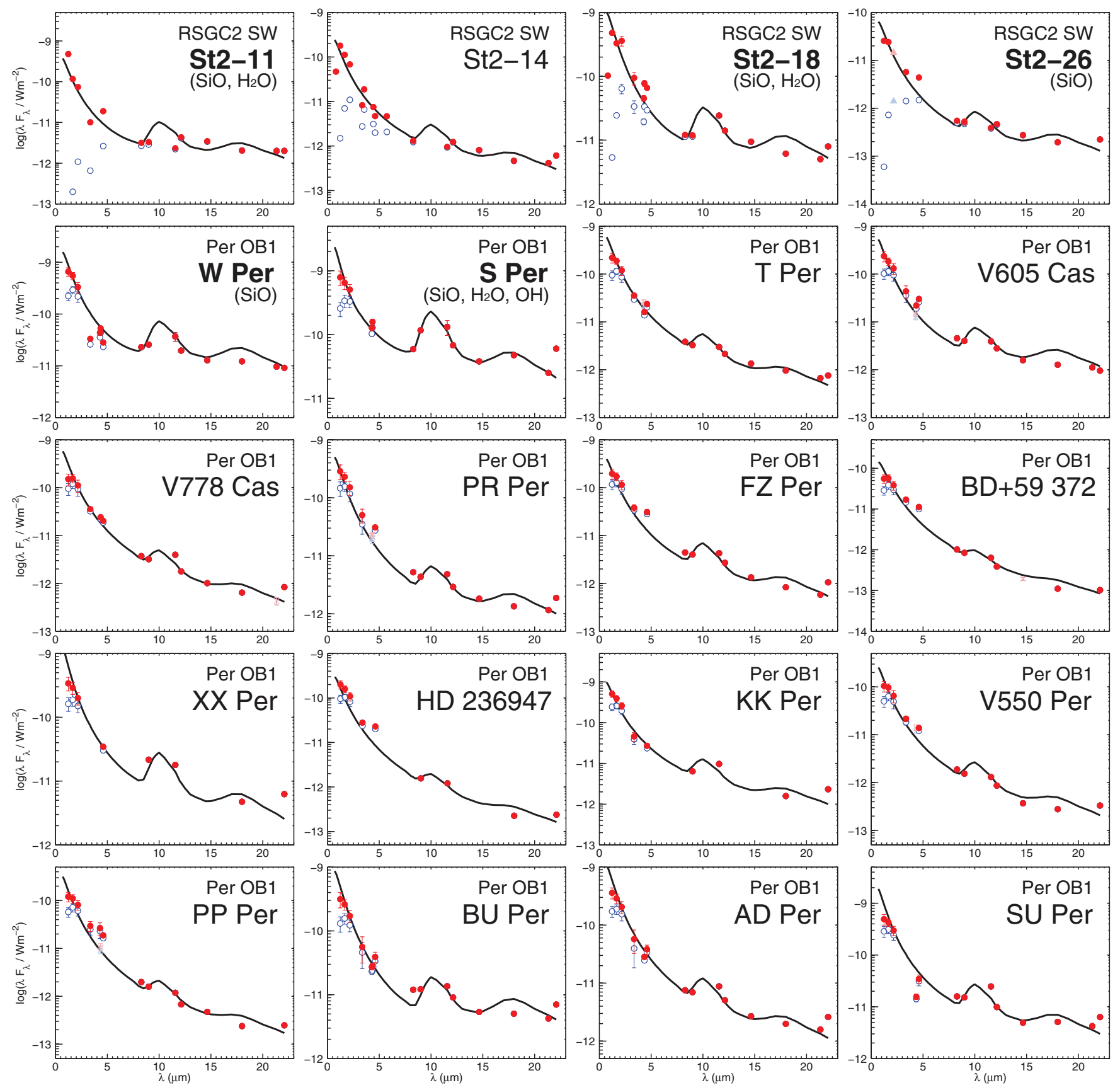

Figure 9. (Continued) 

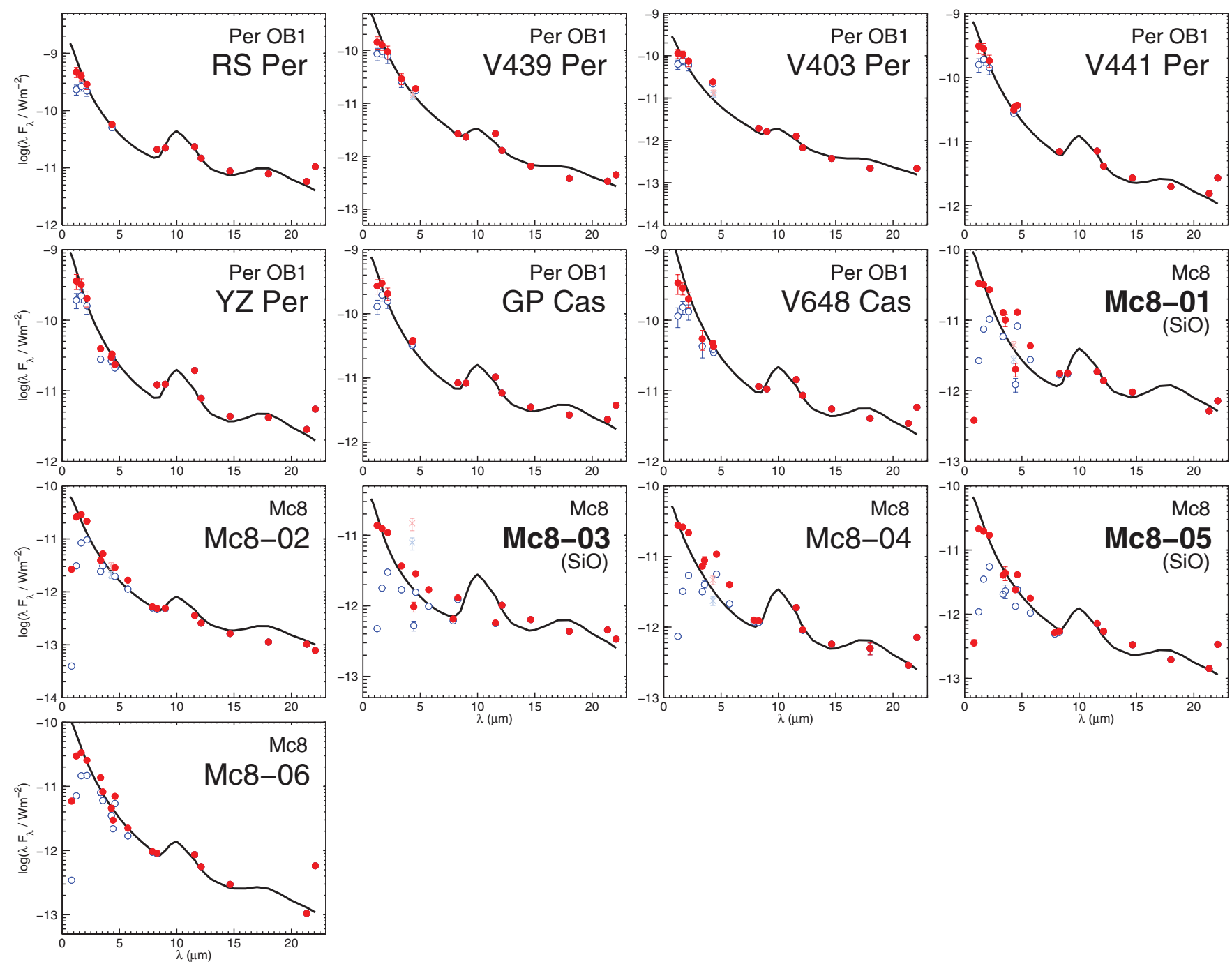

Figure 9. (Continued) 


\section{REFERENCES}

Alexander, M. J., Kobulnicky, H. A., Clemens, D. P., et al. 2009, AJ, 137, 4824 An, D., Terndrup, D. M., Pinsonneault, M. H., et al. 2007, ApJ, 655, 233

Asaki, Y., Deguchi, S., Imai, H., et al. 2010, ApJ, 721, 267

Boboltz, D. A., \& Claussen, M. J. 2004, ApJ, 608, 480

Borgman, J., Koornneef, J., \& Slingerland, J. 1970, A\&A, 4, 248

Brandner, W., Clark, J. S., Stolte, A., et al. 2008, A\&A, 478, 137

Chen, P.-S., \& Gao, Y.-F. 2002, Chinese J. Astron. Astrophys., 2, 169

Cho, S.-H., Kaifu, N., \& Ukita, N. 1996, A\&AS, 115, 117

Choi, Y. K., Hirota, T., Honma, M., et al. 2008, PASJ, 60, 1007

Clark, J. S., Negueruela, I., Crowther, P. A., \& Goodwin, S. P. 2005, A\&A, 434, 949

Clark, J. S., Negueruela, I., Davies, B., et al. 2009, A\&A, 498, 109

Crowther, P. A., Hadfield, L. J., Clark, J. S., et al. 2006, MNRAS, 372, 1407

Crowther, P. A., Hadfield, L. J., Clark, J. S., Negueruela, I., \& Vacca, W. D. 2008, MNRAS, 385, 544

Davies, B., Figer, D. F., Kudritzki, R.-P., et al. 2007, ApJ, 671, 781

Davies, B., Figer, D. F., Law, C. J., et al. 2008, ApJ, 676, 1016

Deguchi, S., Nakada, Y., \& Forster, J. R. 1989, MNRAS, 239, 825

Deguchi, S., Nakashima, J., Zhang, Y., et al. 2010, PASJ, 62, 391

Deul, E., Epchtein, N., \& Borsenberger, J. 1995, Information and On-line Data in Astronomy, Vol. 203, 73

Dollery, M. E., Gaylard, M. J., \& Cohen, R. J. 1987, IAU Symp. 122, 1612 MHz Observations of Southern IRAS Sources (Cambridge: Cambridge Univ. Press), 215

Egan, M. P., Price, S. D., Kraemer, K. E., et al. 2003, VizieR Online Data Catalog, 5114, 0

Figer, D. F., MacKenty, J. W., Robberto, M., et al. 2006, ApJ, 643, 1166

Fouque, P., Le Bertre, T., Epchtein, N., Guglielmo, F., \& Kerschbaum, F. 1992, A\&AS, 93, 151

Haikala, L. K., Nyman, L.-Å., \& Forsstrom, V. 1994, A\&A Suppl. Ser., 103, 107

Humphreys, R. M. 1970, ApJ, 160, 1149

Henry, R. B. C., \& Worthey, G. 1999, PASP, 111, 919

Indebetouw, R., Mathis, J. S., Babler, B. L., et al. 2005, ApJ, 619, 931

Ishihara, D., Onaka, T., Kataza, H., et al. 2010, A\&A, 514, A1

Ivezić, Z., Nenkova, M., \& Elitzur, M. 1999, User Manual for DUSTY, University of Kentucky Internal Report

Jewell, P. R., Batrla, W., Walmsley, C. M., \& Wilson, T. L. 1984, A\&A, 130, L1

Jiang, B. W., Deguchi, S., \& Ramesh, B. 1999, PASJ, 51, 95

Jiang, B. W., Deguchi, S., Yamamura, I., et al. 1996, ApJS, 106, 463

Josselin, E., \& Plez, B. 2005, in High Resolution IR Spectroscopy in Astronomy, ed. U. Käufl et al., ESO Astrophysics Symposia, 405

Kamohara, R., Deguchi, S., Miyoshi, M., \& Shen, Z.-Q. 2005, PASJ, 57, 341

Kothes, R., \& Dougherty, S. M. 2007, A\&A, 468, 993

Le Sidaner, P., \& Le Bertre, T. 1996, A\&A, 314, 896
Levesque, E. M. 2010, New Astron. Rev., 54, 1

Levesque, E. M., Massey, P., Olsen, K. A., et al. 2005, ApJ, 628, 973

Luna, A., Mayya, Y. D., Carrasco, L., Rodríguez-Merino, L. H., \& Bronfman, L. 2009, RevMexAA, 37, 32

Mathis, J. S., Rumpl, W., \& Nordsieck, K. H. 1977, ApJ, 217, 425

Mauron, N., \& Josselin, E. 2011, A\&A, 526, A156

Mengel, S., \& Tacconi-Garman, L. E. 2007, A\&A, 466, 151

Mengel, S., \& Tacconi-Garman, L. E. 2009, Ap\&SS, 324, 321

Messineo, M., Habing, H. J., Sjouwerman, L. O., Omont, A., \& Menten, K. M. 2002, A\&A, 393, 115

Messineo, M., Menten, K. M., Churchwell, E., \& Habing, H. 2012, A\&A, 537, A10

Nakashima, J., \& Deguchi, S. 2006, ApJ, 647, L139

Nakashima, J., \& Deguchi, S. 2007, ApJ, 669, 446

Negueruela, I., Clark, J. S., \& Ritchie, B. W. 2010a, A\&A, 516, A78

Negueruela, I., Gonzalez-Fernandez, C., Marco, A., \& Clark, J. S. 2011, A\&A, 528, A59

Negueruela, I., Gonzalez-Fernandez, C., Marco, A., Clark, J. S., \& MartinezNunez, S. 2010b, A\&A, 513, A74

Nishiyama, S., Nagata, T., Kusakabe, N., et al. 2006, ApJ, 638, 839

Ossenkopf, V., Henning, Th., \& Mathis, J. S. 1992, A\&A, 261, 567

Palagi, F., Cesaroni, R., Comoretto, G., Felli, M., \& Natale, V. 1993, A\&AS, 101,153

Pashchenko, M. I., Rudnitskij, G. M., Samodurov, V. A., \& Tolmachev, A. M. 2006, Astron. Astrophys. Trans., 25, 399

Perryman, M. A. C., Brown, A. G. A., Lebreton, Y., et al. 1998, A\&A, 331, 81

Reach, W. T., Megeath, S. T., Cohen, M., et al. 2005, PASP, 117, 978

Reid, M. J., Menten, K. M., Zheng, X. W., et al. 2009, ApJ, 700, 137

Sault, R. J., Teuben, P. J., \& Wright, M. C. H. 1995, in ASP Conf. Ser. 77, Astronomical Data Analysis Software and Systems IV, ed. R. A. Shaw, H. E. Payne, \& J. J. E. Hayes (San Francisco, CA: ASP), 433

Scholz, M., \& Wood, P. R. 2000, A\&A, 362, 1065

Skrutskie, M. F., Cutri, R. M., Stiening, R., et al. 2006, AJ, 131, 1163

Stothers, R. B., \& Chin, C-W. 1999, ApJ, 522, 960

Su, J. B., Shen, Z.-Q., Chen, X., Yi, Jiyune, Jiang, D. R., \& Yun, Y. J. 2012, ApJ, 754,47

Szymczak, M., Woolak, P., Gérard, E., \& Richards, A. M. S. 2010, A\&A, 524, A99

Takaba, H., Iwata, T., Miyaji, T., \& Deguchi, S. 2001, PASJ, 53, 517

Verheyen, L., Messineo, M., \& Menten, K. M. 2012, A\&A, 541, A36

Werner, M. W., Roellig, T. L., Low, F. J., et al. 2004, ApJS, 154, 1

Wilson, W. J., \& Barrett, A. H. 1972, A\&A, 17, 385

Wright, E. L., Eisenhardt, P. R. M., Mainzer, A. K., et al. 2010, AJ, 140,1868

Zhang, B., Reid, M. J., Menten, K. M., \& Zheng, X. W. 2012a, ApJ, 744, 23

Zhang, B., Reid, M. J., Menten, K. M., Zheng, X. W., \& Brunthaler, A. 2012b, A\&A, 544, A42 